\title{
Cdc42-interacting protein-4 functionally links actin and microtubule networks at the cytolytic NK cell immunological synapse
}

\author{
Pinaki P. Banerjee, ${ }^{1}$ Rahul Pandey, ${ }^{1}$ Rena Zheng, ${ }^{1}$ Megan M. Suhoski, ${ }^{2}$ \\ Linda Monaco-Shawver, ${ }^{1}$ and Jordan S. Orange ${ }^{1,2}$
}

'Children's Hospital of Philadelphia, Division of Immunology, Abramson Research Center, Philadelphia, PA 19104

${ }^{2}$ Immunology Graduate Group, University of Pennsylvania School of Medicine, Philadelphia, PA 19104

\begin{abstract}
An essential function of the immunological synapse (IS) is directed secretion. NK cells are especially adept at this activity, as they direct lytic granules to the synapse for secretion, which enables cytotoxicity and facilitates host defense. This initially requires rearrangement of the actin cytoskeleton and, subsequently, microtubule-dependent trafficking of the lytic granules. As these two steps are sequential, specific linkages between them are likely to serve as critical regulators of cytotoxicity. We studied Cdc42-interacting protein-4 (CIP4), which constitutively interacts with tubulin and microtubules but focuses to the microtubule organizing center (MTOC) after NK cell activation, when it is able to associate with Wiskott-Aldrich syndrome protein (WASp) and the actin filament-rich IS. WASp deficiency, overexpression of CIP4, or parts of CIP4 interfere with this union and block normal CIP4 localization, MTOC polarization to the IS, and cytotoxicity. Reduction of endogenous CIP4 expression using small interfering RNA similarly inhibits MTOC polarization and cytotoxic activity but does not impair actin filament accumulation at the IS, or Cdc42 activation. Thus, CIP4 is an important cytoskeletal adaptor that functions after filamentous actin accumulation and Cdc42 activation to enable MTOC polarization and NK cell cytotoxicity.
\end{abstract}

\section{CORRESPONDENCE}

Jordan S. Orange:

Orange@mail.med.upenn.edu

Abbreviations used: CIP4, Cdc42-interacting protein-4; DIC, differential interference contrast; F-actin, filamentous actin; FCH, Fes/CIP4 homology; IS, immunological synapse; MFI, mean fluorescence intensity; $\mathrm{mIgG}$, mouse isotype-matched $\mathrm{mAb}$ IgG; MTOC, microtubule organizing center; $\mathrm{SH} 3, \mathrm{Src}$ homology 3; siRNA, small interfering RNA; SMAC, supramolecular activation cluster; WAS, Wiskott-Aldrich syndrome; WASp, WAS protein.
The immunological synapse (IS) defines the interface formed between an immune cell and the cell with which it is interacting. It was originally defined in $\mathrm{T}$ cells but has subsequently been characterized in many other types of immune cells. There are several proposed functions for the IS that are likely to vary depending on the cell type. These range from signal generation to termination of effector function, but their relative importance has been debated (1). One of the clearest and first appreciated functions of the IS, however, is directed secretion $(2,3)$. Directed secretion enables precise control in elaborating cell contents onto a specific target cell. This may be especially useful if the secreted components have the potential to harm cells, as in cytotoxicity. In this case, directed secretion serves the purpose of delivering lytic molecules to a target cell while protecting neighboring cells.

Directed secretion requires numerous cell surface and intracellular alterations that facili-

$\overline{\text { The online version of this article contains supplemental material. }}$ tate the movement of secretory components to the IS. The polarization of secretory machinery in cytotoxic cells, such as NK cells, toward a target cell has long been appreciated (4). The process of directed secretion for cytotoxicity culminates with the release of lytic granules, which are secretory lysosomes containing perforin and granzymes (5) onto the target cell. This is required for host defense, as at least three human genetic defects that block this process (mutations in the genes encoding Rab27a, Munc13-4, and syntaxin-11) result in impaired cytotoxicity and susceptibility to viral infection (6-8).

To enable lytic granule release, these organelles polarize to the IS after a cytolytic cell engages a susceptible target cell. Lytic granules can move along microtubules in a kinesindependent manner in vitro (9), and microtubule integrity is required for cytotoxic activity (4). We have been studying the IS and polarization of lytic granules in human NK cells and have found that their movement to the IS depends on microtubule function (10). Polarization of 
lytic granules to the IS, however, is a relatively late step in the formation of the IS and requires several preceding steps, including reorganization of the actin cytoskeleton, clustering of cell-surface receptors, and polarization of the microtubule organizing center (MTOC). Movement of the MTOC to the IS in CTLs is essential for lytic granule delivery and release (11), and NK cells in which the MTOC does not polarize are unable to mediate cytotoxicity $(12,13)$. In $\mathrm{T}$ cells, polarization of the MTOC requires the action of the Cdc42 GTPase (14), and in NK cells it is dependent on activationinduced signaling (13). Although Cdc42 activity is also required for normal filamentous actin (F-actin) accumulation at the IS $(14,15)$, microtubule integrity is not. In NK cells, the F-actin-rich supramolecular activation cluster (SMAC) forms normally after microtubule depolymerization, but SMAC formation and lytic granule polarization are both blocked by inhibition of actin function (10). This suggests that F-actin reorganization in the SMAC is an early and upstream step in the process of NK cell IS formation and function. Similarly, NK cells from patients with Wiskott-Aldrich syndrome (WAS) who lack the WAS protein (WASp), which is required for F-actin reorganization, have reduced F-actin in the SMAC and decreased polarization of lytic granules (16). Although WASp-dependent F-actin reorganization is an essential prerequisite, reorientation of the MTOC to the SMAC is a likely critical late step in cytotoxicity. In particular, the ability to link these two steps involving the reorganized actin cytoskeleton and microtubule network in NK cells could help facilitate the directed secretion of lytic granules. A specific protein with these capabilities in cytotoxic cells, however, has not been demonstrated, but would define an important regulatory step in formation of the cytolytic IS and cytotoxicity.

We have studied Cdc42-interacting protein-4 (CIP4) as a potential candidate to link linear stages in cytolytic-directed secretion. CIP4 was discovered for its ability to interact with Cdc42 and with WASp through an Src homology 3 (SH3) domain $(17,18)$. It also contains a Fes/CIP4 homology (FCH) domain, which enables it to interact with tubulin, and may be relevant to podosome formation in macrophages (19). In this study, we have determined that appropriate expression of CIP4 in NK cells is required for MTOC polarization and cytotoxicity. We show that CIP4 biochemically and spatially unites $\mathrm{F}$-actin and microtubule networks in activated NK cells but functions downstream of $\mathrm{Cdc} 42$ activation. Thus, we propose that CIP4 is an important functional linkage between the SMAC and MTOC polarization to obtain secretory cytolytic effector function.

\section{RESULTS \\ CIP4 is expressed in NK cells and localizes with the MTOC to the IS after NK cell activation}

To determine if CIP4 could serve a role in NK cells, we initially documented its expression. Using overlapping primer sets that encompassed the complete coding sequence, a CIP4 message was identified among cDNA in all immortalized NK cell lines tested, as well as in ex vivo NK cells (Fig. 1 A). Sequence analysis of amplified product confirmed consensus in all NK cell samples. CIP4 was also detected by Western blotting among total lysates using mAb anti-CIP4 in NK cell lines and ex vivo NK cells, albeit at levels lower than those found in fibroblasts (not depicted) (Fig. 1 B). Levels of WASp were more similar among the different NK cells and, unlike CIP4, were proportional to $\alpha$-tubulin, which is shown as a protein loading control. To more accurately determine the relative levels of CIP4 in NK cells, intracellular FACS for CIP4 was developed (Fig. 1 C). Relative to isotype control, CIP4 content was uniform in the different NK cell lines but was lower in NK cells among ex vivo PBMCs (Fig. 1 D). This result was consistent with the smaller size of ex vivo NK cells compared with the NK cell lines (unpublished data).

CIP4 has important associations with WASp and microtubules in nonimmune cells, as well as in macrophage podosomes $(18,19)$. Because we have identified critical roles for WASp and microtubules in the polarization of lytic granules to the IS in NK cells $(10,16)$, we wanted to study CIP4 relative to the IS. As an initial approach, we used YTS NK cells stably expressing a CD2-GFP fusion molecule, as CD2 clustering serves as an early marker of the IS in NK cells (10). Like certain subpopulations of ex vivo NK cells, YTS cells can be induced to mediate cytotoxicity after ligation of CD28 (20, 21). Because K562 cells do not express CD80 or CD86, they are not susceptible to lysis by YTS cells (unpublished data). To use K562 cells as a susceptible target for YTS cells that would induce a cytolytic IS, the K562 cells were stably transduced with CD86 (KT86 cell line). KT86 cells were killed by YTS cells, as measured in ${ }^{51} \mathrm{Cr}$ release assays, and provide the advantage of isolating a specific activation pathway in YTS cells. Direct comparison of KT86 and K562 cells also affords a comparison of cytolytic and noncytolytic interactions.

Using YTS CD2-GFP cells, the localization of CIP4 relative to the IS and to the MTOC was determined by confocal microscopy in cytolytic (Fig. 2, A-F) and noncytolytic (Fig. 2, $\mathrm{G}-\mathrm{L}$ ) conjugates. In this setting, the MTOC was visualized as an accumulation of $\alpha$-tubulin and was defined using an intensity and size threshold, or as the region containing the centrosomal protein pericentrin (Fig. 2, C and I). In cytolytic conjugates formed between YTS and KT86 cells, the IS as defined by the presence of accumulated CD2-GFP contained a polarized tubulin-defined MTOC, and accumulated CIP4 (Fig. 2, A-F). The noncytolytic synapse also contained accumulated CD2-GFP but had reduced MTOC polarization and accumulation of CIP4 (Fig. 2, G-L). In experiments where the nucleus was visualized, the MTOC and CIP4 could be found between the nucleus and the IS in YTS CD2-GFP cells engaged in cytolytic, but not in noncytolytic, conjugates (Fig. S1, available at http://www.jem.org/cgi/content/full/ jem.20061893/DC1). Assessment of multiple conjugates over separate experiments demonstrated that although CD2-GFP accumulation at the IS was slightly decreased in noncytolytic compared with cytolytic synapses (by 14\%), polarization of 

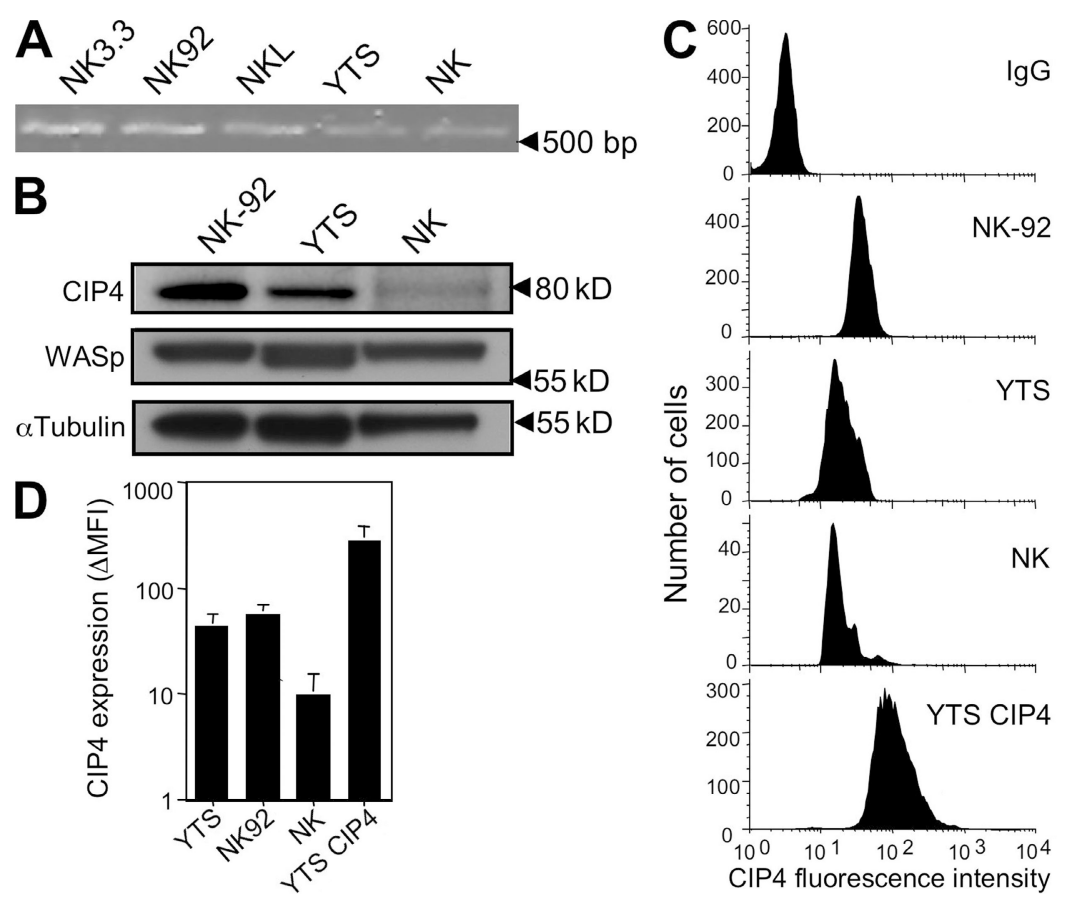

Figure 1. CIP4 expression in NK cells. (A) RT-PCR for CIP4 message in NK cell lines and ex vivo NK cells. (B) Western blot (10 $\mu g$ of protein per lane) for CIP4 in NK92, YTS, and ex vivo NK cells, as well as WASp and $\alpha$-tubulin after stripping and reprobing membranes. (C) Intracellular CIP4 FACS using CIP4 mAb or IgG clone MOPC21 (in YTS cells as a specificity control, which was comparable with IgG control for the other cell types). Ex vivo NK cells were identified by FACS in total PBMCs by costaining for CD3 and CD56 and gating on CD3- ${ }^{-}, C D_{5} 6^{+}$lymphocytes (NK). (D) The increase in CIP4 mean fluorescence intensity (MFI) over control IgG detected by FACS for YTS, NK92, ex vivo NK, and CIP4 YTS cells in three experiments and with six different donors of ex vivo cells is shown. IgG MFI was determined in parallel with each repeated assessment of CIP4. Error bars represent the SD.

the MTOC and accumulation of CIP4 were substantially reduced (by 72\%; Fig. 2 M). To assess MTOC polarization in this system more definitively, the mean distance of the tubulinor pericentrin-defined MTOC from the IS was also measured in cytolytic and noncytolytic conjugates. The MTOC was significantly closer to the IS in cytolytic conjugates (Fig. $2 \mathrm{~N}$ ), consistent with the observed increase in MTOC polarization and with the role of the MTOC in facilitating lytic granule delivery (11).

To quantitatively assess colocalization between CIP4 and the MTOC, the area occupied by the different fluorescent intensities within the YTS cells was measured. The area of the tubulin-defined MTOC was distinguished from the fluorescence of unaccumulated $\alpha$-tubulin and individual microtubules, but in the plane of the MTOC contains the majority of the $\alpha$-tubulin fluorescent area (Fig. 2 O). The area occupied by $\alpha$-tubulin, the tubulin-defined MTOC, or CIP4 did not differ between YTS cells in cytolytic and noncytolytic conjugates (Fig. 2 O). In contrast, the colocalization between $\alpha$-tubulin, or the tubulin-defined MTOC, and CIP4 was increased in cytolytic conjugates (Fig. 2 P). Similarly, the colocalization between CIP4 and the tubulin-defined MTOC or $\alpha$-tubulin was also higher in cytolytic conjugates. Because the area of CIP4 did not increase in cytolytic YTS cells, this suggested that the existing CIP4 focused to the MTOC during the cytolytic process. As differences in CIP4 colocalization between $\alpha$-tubulin and the tubulin-defined MTOC were not significant $(P=0.22)$, further analyses focused on the tubulin-defined MTOC.

Colocalization analyses were also performed using a pericentrin-defined MTOC. There was 75-97\% colocalization between pericentrin and the tubulin-defined MTOC but only $45 \%$ colocalization between the tubulin-defined MTOC and the pericentrin-defined MTOC (Fig. $2 \mathrm{Q}$ ). Thus, the area occupied by the pericentrin-defined MTOC was less than the tubulin-defined MTOC. Colocalization between the pericentrin-defined MTOC and CIP4, however, was greater in cytolytic conjugates than the colocalization between CIP4 and the tubulin-defined MTOC. This difference was not found in noncytolytic conjugates, as CIP4 colocalization with the MTOC was less (note MTOC enlargement in Fig. S2, available at http://www.jem.org/cgi/content/full/ jem.20061893/DC1) and, thus, is consistent with the centrosomal focusing of CIP4 in cytolytic conjugates. As an alternative to these whole-cell area and intensity cell-based colocalization analyses, the colocalization within a constant size region around the MTOC was also performed and defined a similarly increased colocalization between the MTOC and CIP4 in YTS CD2-GFP cells engaged in cytolytic versus noncytolytic conjugates (Fig. S2).

Because CIP4 could have the potential to facilitate interaction between the MTOC and F-actin during formation of 

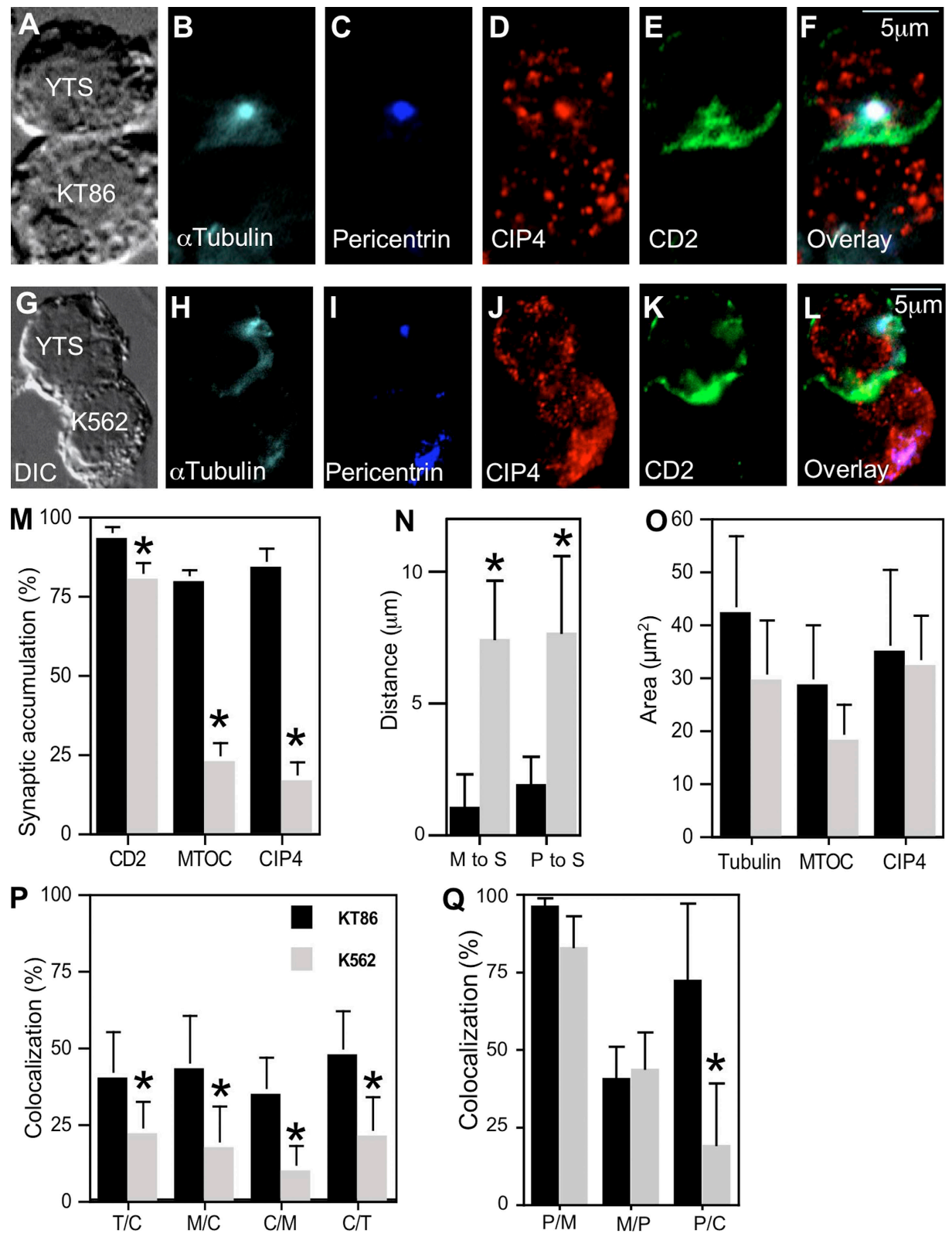

Figure 2. Accumulation of CIP4 with the MTOC at the cytolytic but not noncytolytic IS. The cytolytic IS (A-F) between a YTS CD2-GFP cell and a KT86 cell, and a noncytolytic IS (G-L) between a YTS CD2-GFP cell and a K562 cell, as viewed by DIC microscopy (A and G), as well as confocal microscopy showing fluorescence for $\alpha$-tubulin ( $\mathrm{B}$ and $\mathrm{H}$ ), pericentrin ( $\mathrm{C}$ and I), CIP4 (D and J), CD2-GFP (E and K), and an overlay (F and L). Although an X-y plane was selected in the $z$ axis to highlight the MTOC, disproportionate accumulation of CIP4 in other x-y planes was not found. (M) Accumulation of CD2, the tubulin-defined MTOC, or CIP4 in > 200 conjugates over four experiments at the cytolytic (black bars) and noncytolytic (gray bars) IS. (N) Mean distance of the tubulin- (M to S) or pericentrin-defined (P to S) MTOC from the IS in YTS cells conjugated with KT86 (black bars) or K562 (gray bars) target cells is shown. Distances were greater in noncytolytic conjugates. (0) The area of $\alpha$-tubulin, the MTOC, and CIP4 was measured in the $x-y$ plane containing the MTOC in YTS cells conjugated with KT86 (black bars) or K562 (gray bars) target cells. The MTOC was defined as the region consisting of accumulated $\alpha$-tubulin through the exclusion of nonaccumulated $\alpha$-tubulin using a size and intensity threshold. (P) The percent colocalization calculated among areas is shown for the percentage of $\alpha$-tubulin that colocalized with CIP4 (T/C), the MTOC that colocalized with CIP4 (M/C), CIP4 that colocalized with the MTOC $(\mathrm{C} / \mathrm{M})$, and CIP4 that colocalized with $\alpha$-tubulin (C/T). Significant differences between the cytolytic (black bars) and noncytolytic (gray bars) synapses are noted. (0) In YTS cells engaged in cytolytic (black bars) and noncytolytic (gray bars) conjugates, the mean percentage of the pericentrin-defined MTOC that colocalized with the $\alpha$-tubulin-defined MTOC (P/M), the percentage of the $\alpha$-tubulin-defined MTOC that colocalized with the pericentrin-defined MTOC (M/P), and the percentage of the pericentrin-defined MTOC that colocalized with CIP4 (P/C) are shown. Error bars represent the SD. ${ }^{*}, \mathrm{P}<0.01$. 
the mature lytic IS, F-actin was evaluated relative to CIP4 and the MTOC after their increased association at the cytolytic IS. NK92 cells were studied in addition to YTS cells to ensure that the findings were not unique to YTS (Fig. 3, A-L). K562 cells are susceptible to NK92 cell-mediated lysis and, thus, were used as physiologic target cells for the NK92 cells. To discern the NK cell from the target cell, YTS cells expressing GFP were conjugated with KT86 cells. Similarly, K562 cells expressing GFP were used to make conjugates with NK92 cells. In each case, conjugates were selected that contained one $\mathrm{GFP}^{+}$cell paired with one $\mathrm{GFP}^{-}$cell (Fig. 3,
$\mathrm{B}$ and $\mathrm{H}) . \mathrm{F}$-actin, the $\alpha$-tubulin-defined MTOC, and CIP4 accumulated at the majority of synapses in both YTS (Fig. 3, C-F) and NK92 cells (Fig. 3, I-L), though the F-actin accumulation was a more consistent feature (Fig. $3 \mathrm{M}$ ). The area occupied by F-actin, the MTOC, and CIP4 was equivalent in the two NK cell types (Fig. $3 \mathrm{~N}$ ), as was the pattern of colocalization. Specifically, slightly more than half of the total MTOC area contained CIP4 and slightly less than half of the total CIP4 area contained the MTOC (Fig. 3 O). In contrast, approximately one quarter of the total F-actin area contained CIP4 and only one tenth of the total CIP4 area contained
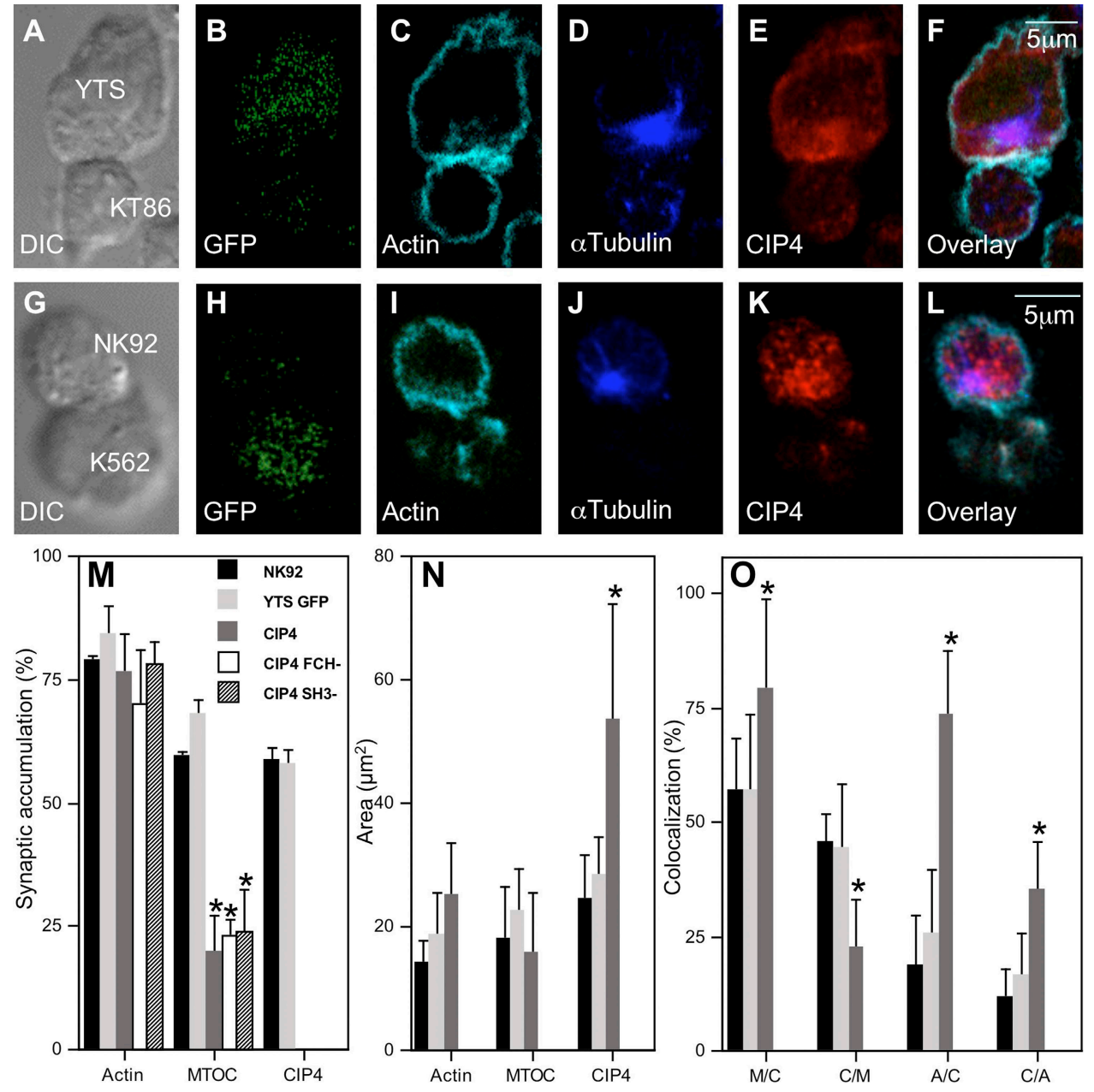

Figure 3. Colocalization of CIP4 with the MTOC and accumulated F-actin at the cytolytic IS. Cytolytic conjugates between a YTS GFP cell and a KT86 cell (A-F), and an NK92 cell and a K562 GFP cell (G-L). DIC images (A and G) and GFP expression in the YTS cell (B), or in the K562 cell (H), distinguish the NK cell from the target cell. Confocal microscopy for F-actin ( $C$ and I), $\alpha$-tubulin ( $D$ and J), CIP4 (E and K), and an overlay (F and L) are shown. (M) Molecular accumulations in NK cells from $\geq 150$ conjugates over at least three experiments between NK92 and K562 cells, YTS GFP and KT86 cells, YTS CIP4 and KT86 cells, CIP4 FCH ${ }^{-}$and KT86 cells, and CIP4 SH3 ${ }^{-}$and KT86 cells. CIP4 accumulation was not determined for the CIP4-overexpressing cells, as it was diffuse. (N) The total area of F-actin, the tubulin-defined MTOC, and CIP4 measured in $\geq 15$ conjugated NK cells over three experiments between NK92 and K562 cells (black bars), YTS GFP and KT86 cells (light gray bars), and YTS CIP4 and KT86 cells (dark gray bars). CIP4 mutants were not detected with anti-CIP4 mAb. (0) The percent colocalization among the areas in $\geq 15$ conjugated NK cells over three experiments between NK92 and K562 cells (black bars), YTS GFP and KT86 cells (light gray bars), and YTS CIP4 and KT86 cells (dark gray bars) was calculated to demonstrate the percentages of the MTOC colocalized with CIP4 (M/C), of the CIP4 colocalized with the MTOC (C/M), of the F-actin colocalized with CIP4 (A/C), and of the CIP4 colocalized F-actin (C/A). Differences between YTS GFP and NK92 cells were not significant, but those between YTS CIP4 and YTS GFP cells were. Error bars represent the SD. ${ }^{*}, \mathrm{P}<0.01$. 
F-actin. Thus, at the cytolytic IS, CIP4 shared space with a substantial portion of the MTOC and a small amount of F-actin. This raised the possibility that CIP4 could serve a functionally important role in linking the MTOC to a critical region of F-actin at the IS.

CIP4 or truncated CIP4 overexpression blocks NK cell cytotoxicity by altering cytoskeletal associations and preventing MTOC polarization to the IS

To investigate the functional importance of CIP4 in cytotoxicity and at the cytolytic IS, we initially took an overexpression approach. We hypothesized that if CIP4 was to serve a role as an important link between the MTOC and F-actin, unregulating its expression would interfere with its physiological role as a linker. Full-length WT CIP4 cDNA or CIP4 cDNA deleted of the nucleotides encoding the first 117 aa encompassing the $\mathrm{FCH}$ domain $\left(\mathrm{FCH}^{-}\right)$, or encoding the last 128 aa encompassing the $\mathrm{SH} 3$ domain $\left(\mathrm{SH}^{-}\right)$, were cloned into the pMx-IRES-GFP retroviral vector (Fig. 4 A). These vectors were packaged into ecotropic retroviruses and were used to infect YTS cells stably expressing the ecotropic receptor (22). Transduced cells were identified through GFP expression and sorted by FACS to create cultures stably expressing high or low levels (Fig. 4 B). Anti-CIP4 pAb was used in Western blots of lysates of these cells to confirm the presence of CIP4 protein, and the high GFP-expressing cell lines (YTS-CIP4) had the highest levels of CIP4 protein (Fig. 4 C). The low expressing cells still had levels of CIP4 that were higher than endogenous levels. The $\mathrm{pAb}$ failed to recognize the $\mathrm{FCH}^{-}$, and the $\mathrm{mAb}$ failed to recognize either mutant construct (unpublished data). Overexpressed WT CIP4 could be detected using mAbs by intracellular FACS
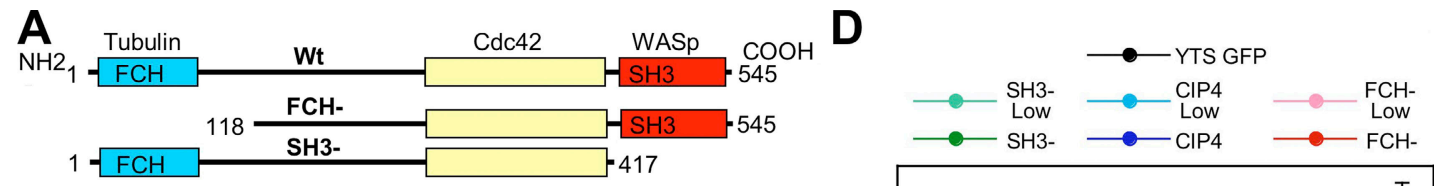

B
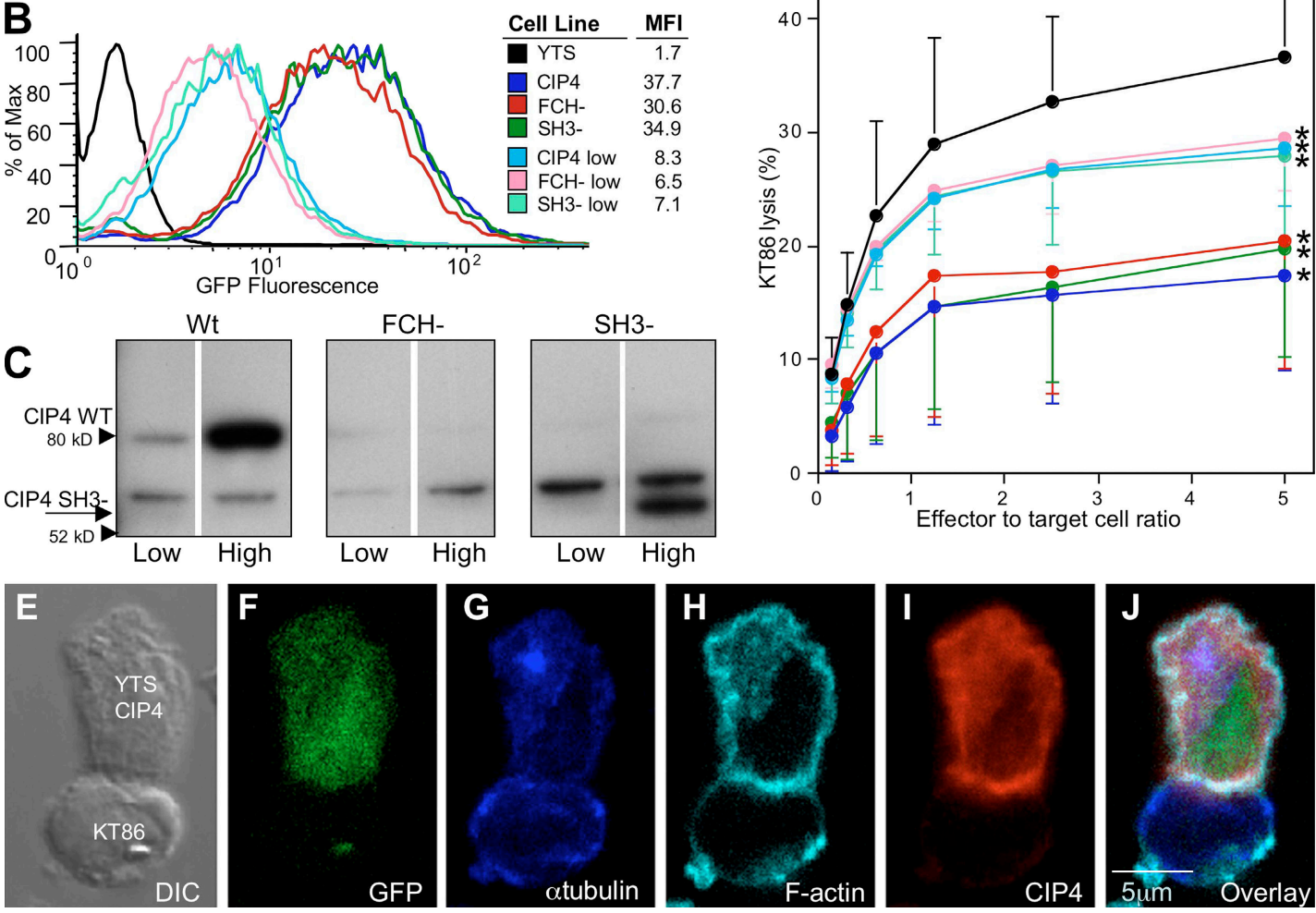

Figure 4. Stable overexpression of CIP4 or CIP4 mutants in NK cells. (A) Schematic of constructs for CIP4 WT (top), $\mathrm{CIP}_{4}$ FCH- (middle), or $\mathrm{SH}^{-}$(bottom) used to generate bicistronic retroviral expression vectors (blue, FCH domain; red, SH3 domain. (B) Transduced YTSeco cells were sorted via FACS into populations with comparable GFP expression (numbers show GFP MFI), and cultures had consistent GFP expression over time. (C) Western blot of CIP4 WT (left), $\mathrm{CIP}_{4} \mathrm{FCH}^{-}$(middle), and $\mathrm{CIP}_{4} \mathrm{SH}^{-}$(right) low and high overexpressing cell lines using anti-CIP4 pAb. The middle band is nonspecific, and the antibody failed to recognize the FCH domain-deleted CIP4. (D) Cytotoxicity of CIP4-overexpressing YTS cells against KT86 target cells. Each point represents the mean of four experiments, and decreased activity was significant $(P<0.01)$ for all effector/target ratios in high, but only at 5:1 and 2.5:1 in low, overexpressing cells. (E and F) A representative conjugate between YTS CIP4 high overexpressing cells (top) and a KT86 target cell (bottom) was evaluated using DIC (E) and by confocal microscopy for GFP expression (F), $\alpha$-tubulin (G), F-actin (H), CIP4 (I), and an overlay (J). 
and was present at substantially higher levels than YTS, NK92, or ex vivo NK cells (Fig. 1, C and D).

Because NK cell cytolytic activity requires the coordinated and sequential function of both actin and microtubules, we determined the impact of CIP4 overexpression by initially measuring cytotoxicity. The lytic activity of all six new cell lines was measured against KT86 target cells and was compared with YTS cells expressing levels of GFP similar to those found in the high CIP4-expressing lines. In contrast to cells expressing GFP alone, cytotoxic function was inhibited by overexpression of the WT or mutant CIP4 constructs (Fig. 4 D). This was not caused by decreased CD28 expression (unpublished data). Inhibition of cytotoxicity by CIP4 overexpression was not absolute but was proportional to the level of transgene expression and did not require the simultaneous presence of the $\mathrm{FCH}$ or $\mathrm{SH} 3$ domains. This result suggested that unregulated expression of CIP4 results in an abnormal role for this protein that interferes with the normal physiological process of cytotoxicity.

To determine how CIP4 overexpression interferes with cytotoxicity, CIP4-overexpressing cells conjugated with KT86 target cells were evaluated by confocal microscopy to study cytoskeletal reorganization and CIP4 localization. YTS cells overexpressing CIP4 were identified in conjugates with KT86 cells through expression of GFP via the IRES in the transduced construct (Fig. 4 F). In cells overexpressing WT CIP4, the tubulin-defined MTOC generally failed to polarize to the IS, but F-actin still accumulated (Fig. 4, G and H). Interestingly, in WT CIP4-overexpressing cells, CIP4 was no longer primarily found with the MTOC but was now found in the cell cortex, as seen by colocalization with F-actin. The decreased polarization of the MTOC to the IS was a consistent feature of CIP4 overexpression even in the absence of the $\mathrm{FCH}$ or SH3 domains, as all overexpressing cells had less than one third the polarization of the tubulin-defined MTOC compared with cells expressing GFP alone (Fig. $3 \mathrm{M}$ ). Similar to the WT CIP4-expressing cells, each of the mutantexpressing lines maintained the ability to accumulate F-actin at the IS (Fig. $3 \mathrm{M}$ and Fig. S3, available at http://www .jem.org/cgi/content/full/jem.20061893/DC1), suggesting that the impact of CIP4 overexpression was downstream of F-actin reorganization. As perforin and lytic granules polarize to the activating NK cell IS with the MTOC $(12,13)$, perforin was evaluated in YTS cells overexpressing each of the CIP4 constructs and did not appear disrupted in its normal proximity to the MTOC (Fig. S3). Thus, the movement of perforin-containing lytic granules relative to the MTOC would not be expected to be impaired by CIP4 overexpression. Instead, these data indicate a more specific effect of CIP4 on MTOC localization.

Because actin accumulation at the IS appeared intact, a potential mechanism by which CIP4 overexpression could interrupt MTOC polarization and cytotoxicity might be by occupying physiological binding sites in the F-actin network. Quantitative analysis of confocal micrographs demonstrated that although the area occupied by CIP4 after overexpression increased twofold (Fig. $3 \mathrm{~N}$ ), the colocalization between F-actin and CIP4 increased threefold (Fig. $3 \mathrm{O}$ ), and a majority of F-actin colocalized with CIP4. There was a slight increase in the area of the tubulin-defined MTOC that colocalized with CIP4, but the relative amount of the CIP4 that colocalized with the MTOC was decreased by $49 \%$. Similarly, the relative amount of CIP4 colocalized with F-actin increased after overexpression, and the CIP4 was almost equally
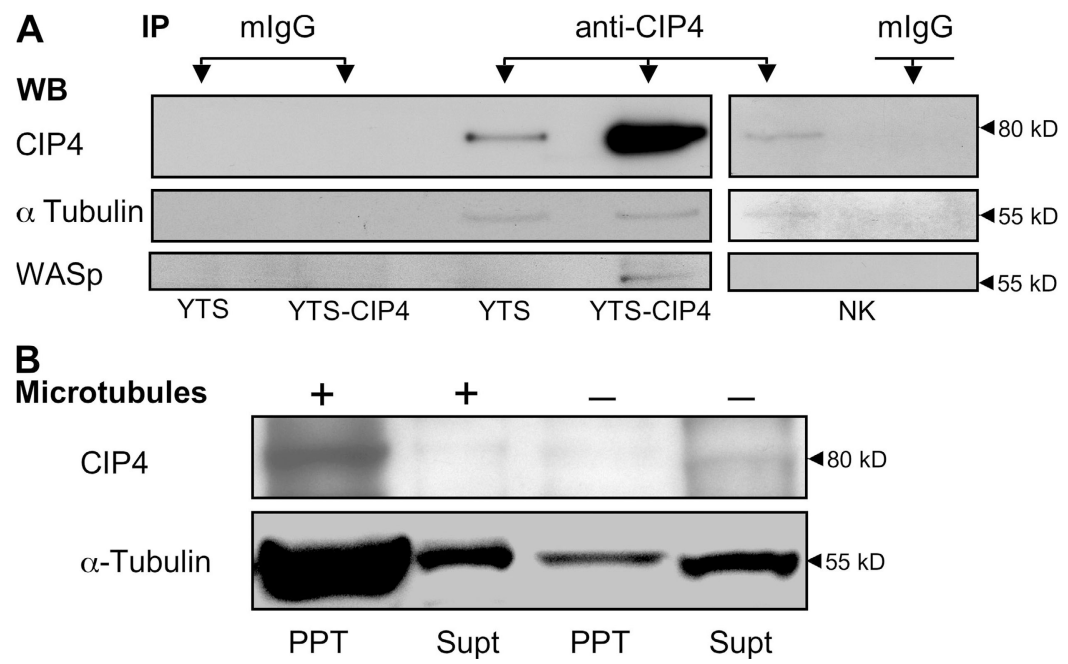

Figure 5. Associations of endogenous and overexpressed CIP4. (A) CIP4 was precipitated from lysates of $2 \times 10^{6}$ YTS CIP4 cells, $2 \times 10^{6}$ parental YTS cells, or $2 \times 10^{7}$ ex vivo NK cells using mAb anti-CIP4. Immunoprecipitation with nonspecific mouse isotype-matched $\mathrm{mAb} \operatorname{lgG}(\mathrm{mlgG})$ is shown as a control. CIP4 was identified in immunoprecipitates by Western blotting using anti-CIP4 mAb (top). Blots were stripped and reprobed for $\alpha$-tubulin (middle) and WASp (bottom; blots represent three to six independent results). (B) YTS cell lysates cleared of nuclei and debris were incubated with or without stabilized microtubules, after which microtubules and associated proteins were precipitated. CIP4 and $\alpha$-tubulin were identified by Western blotting in the supernatant (Supt) and precipitate (PPT). 
divided in colocalization with the MTOC and F-actin. Thus, after overexpression, CIP4 appears to occupy nonphysiologic space in the F-actin cortex, potentially interfering with the normal physiologic role of the CIP4 that is associated with the MTOC.

To confirm this association biochemically, CIP4 was immunoprecipitated from WT CIP4-overexpressing YTS, parental YTS, and ex vivo NK cells. In lysates of the overexpressing cells, CIP4 coimmunoprecipitated with both $\alpha$-tubulin and WASp (as predicted by the microscopy data), whereas in parental YTS and ex vivo NK cells, CIP4 preferentially coimmunoprecipitated with $\alpha$-tubulin (Fig. 5 A). This demonstrates that overexpressed CIP4 was nonphysiologic in its association with WASp but that it had the capacity to link elements that function in the F-actin cortex with $\alpha$-tubulin. To understand if the association between CIP 4 and $\alpha$-tubulin in YTS cells could be indicative of an association with microtubules, YTS lysates were cleared of debris and exposed to stabilized microtubules. Microtubules removed CIP4 from YTS lysates and led to CIP4 concentration in the microtubule precipitate (Fig. 5 B). Collectively, these data show that CIP4 is a microtubule-associated protein in YTS cells but is largely not associated with WASp under resting conditions.
WASp is required for the activation-induced physiologic associations of CIP4

To extend these results to ex vivo NK cells and to evaluate the role for WASp in greater detail, CIP4 was studied in NK cells prepared from healthy donors or a patient with WAS who had no WASp protein. Like YTS and NK92 cells, in conjugates formed between ex vivo NK cells and susceptible target cells, CIP4 and the tubulin-defined MTOC polarized to the IS near the region of accumulated F-actin (Fig. 6, A-E). A similar result was obtained in ex vivo NK cells using a pericentrin-defined MTOC (Fig. S4, available at http:// www.jem.org/cgi/content/full/jem.20061893/DC1). In contrast, when ex vivo NK cells were deficient in WASp, there was reduced $\mathrm{F}$-actin accumulation, as well as reduced polarization of the MTOC and CIP4 (Fig. 6, F-K). In the WAS patient NK cells, the CIP4 appeared to be more diffusely located throughout the cytoplasm and was typically not seen in the cell cortex. Compared with control cells, the total area occupied by F-actin and the MTOC was reduced in WASpatient NK cells (Fig. 6 L). The area occupied by CIP4, however, was equivalent to that found in control cells. As expected, a lower percentage of the CIP4 area in WASpatient cells colocalized with the reduced areas of either F-actin or the MTOC (Fig. $6 \mathrm{M}$ ). Surprisingly, despite the
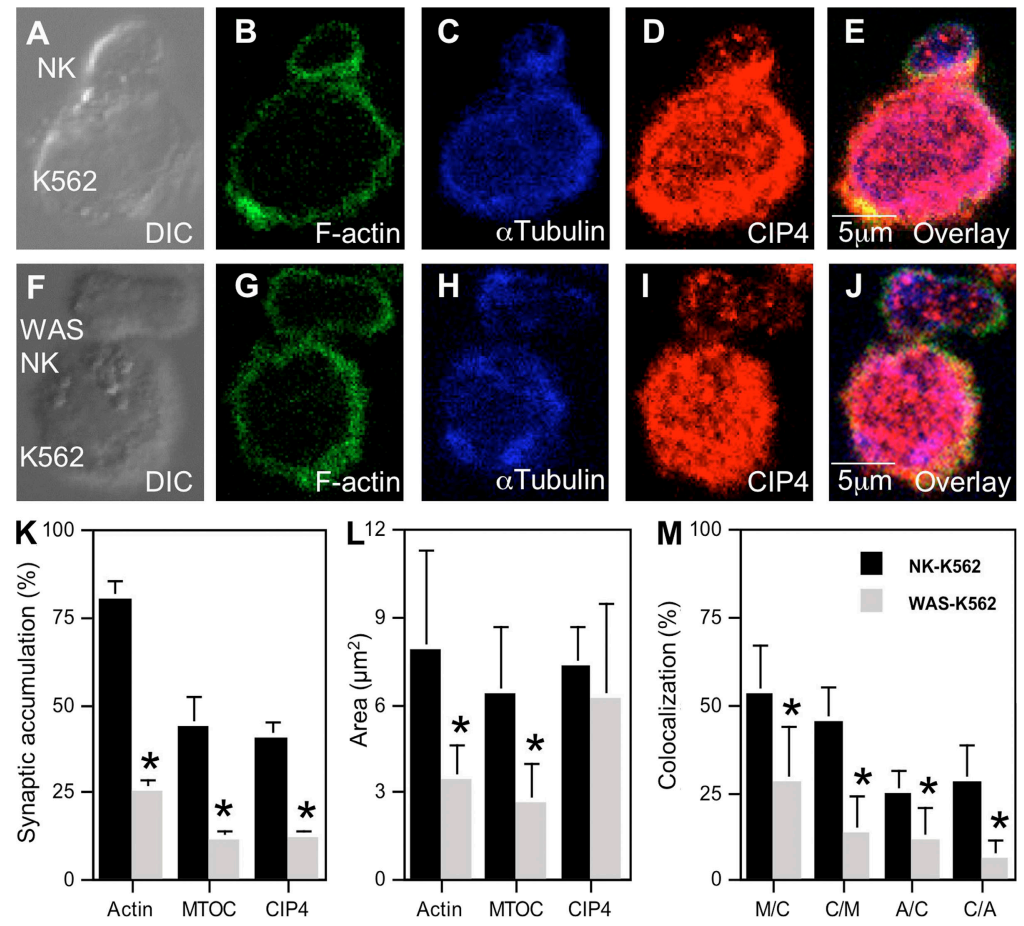

Figure 6. Localization of CIP4 in normal and WASp-deficient ex vivo NK cells. An NK cell (top) and target cell (bottom) in conjugates between a normal donor ex vivo NK cell and a K562 target cell (A-E), as well as a WAS-patient ex vivo NK cell and a K562 target cell (F-J), using DIC microscopy (A and F). F-actin (B and G), $\alpha$-tubulin (C and H), CIP4 (D and I), and an overlay (E and J) were detected using confocal microscopy. (K) Molecular accumulation at the IS in >150 NK cells conjugated with K562 target cells in at least three experiments are shown using normal donor (black bars) or WAS-patient (gray bars) NK cells. (L) The area of F-actin, the $\alpha$-tubulin-defined MTOC, and CIP4 was measured in $\geq 15$ conjugated NK cells over three experiments between K562 target cells and normal donor (black bars) or WAS-patient NK cells (gray bars). (M) The percentage of the MTOC that colocalized with CIP4 (M/C), CIP4 that colocalized with the MTOC (C/M), F-actin that colocalized with CIP4 (A/C), and CIP4 that colocalized with F-actin (C/A) was calculated for the measured areas in the conjugated normal donor and WAS-patient NK cells. Decreases in WAS-patient NK cells were significant. ${ }^{*}, \mathrm{P}<0.01$. 
similar area of CIP4 in WAS-patient cells, the percentage of the MTOC or F-actin that colocalized with CIP4 was less than that found in healthy donor ex vivo NK cells. Thus, WASp was required to enable the normal physiologic localization and associations of CIP4.

\section{The association between CIP4 and WASp is specific to NK cell activation}

Because WASp was required for the physiologic associations of CIP4 but CIP4 only coimmunoprecipitated with WASp after CIP4 overexpression (Fig. 5 A), we hypothesized that NK cell activation enables CIP4 function and, specifically, the association between WASp and CIP4. To evaluate this, we needed to activate NK cells in the absence of target cells, because the target cell can serve as a source of both WASp and CIP4. This would make it impossible to discern whether the molecule being studied was contributed by the effector or the target cell. Furthermore, the presence of target cell protein in a cell lysate may be of a distinct activation state and could thus dilute or affect results. To activate YTS and ex vivo NK cells, activation receptors were clustered by placing cells on antibody-coated surfaces. Anti-CD28 was used for YTS cells and anti-NKp30 was used for ex vivo NK cells, as these antibodies have been shown to trigger cytotoxicity and activation signaling $(21,23)$. Cells were incubated for $30 \mathrm{~min}$ on glass slides (for microscopy) or plastic wells (for biochemical analysis) coated with the antibody. Surfaces coated with either IgG or media were both used as controls. To confirm that the activating signal had an effect on F-actin, the MTOC, and CIP4, cells were evaluated using laser scanning confocal microscopy and scanning through the $\mathrm{x}-\mathrm{z}$ axis to visualize their contact with the antibody. Both YTS cells and ex vivo NK cells demonstrated a preferential accumulation of F-actin at, as well as a polarization of the tubulin-defined MTOC and CIP4 to, the activating antibody-coated surface when compared with the IgG-coated surface (Fig. 7, A-D). The IgG-coated slide, however, did induce some F-actin accumulation in ex vivo NK cells at the glass surface when compared with slides not coated with antibody (unpublished data). This was presumably caused by the Fc receptor present on ex vivo NK cells, which was absent on YTS cells. This is consistent with data demonstrating IS formation without MTOC polarization after CD16 ligation in NK cells (24). Despite this, cells with F-actin, the MTOC, and CIP4 accumulated at the activating $\mathrm{mAb}$-coated glass surface more than doubled in number when compared to the IgG-coated glass surface ( $n \geq 100$ adhered cells over three experiments). The colocalization between the CIP4 and the MTOC also
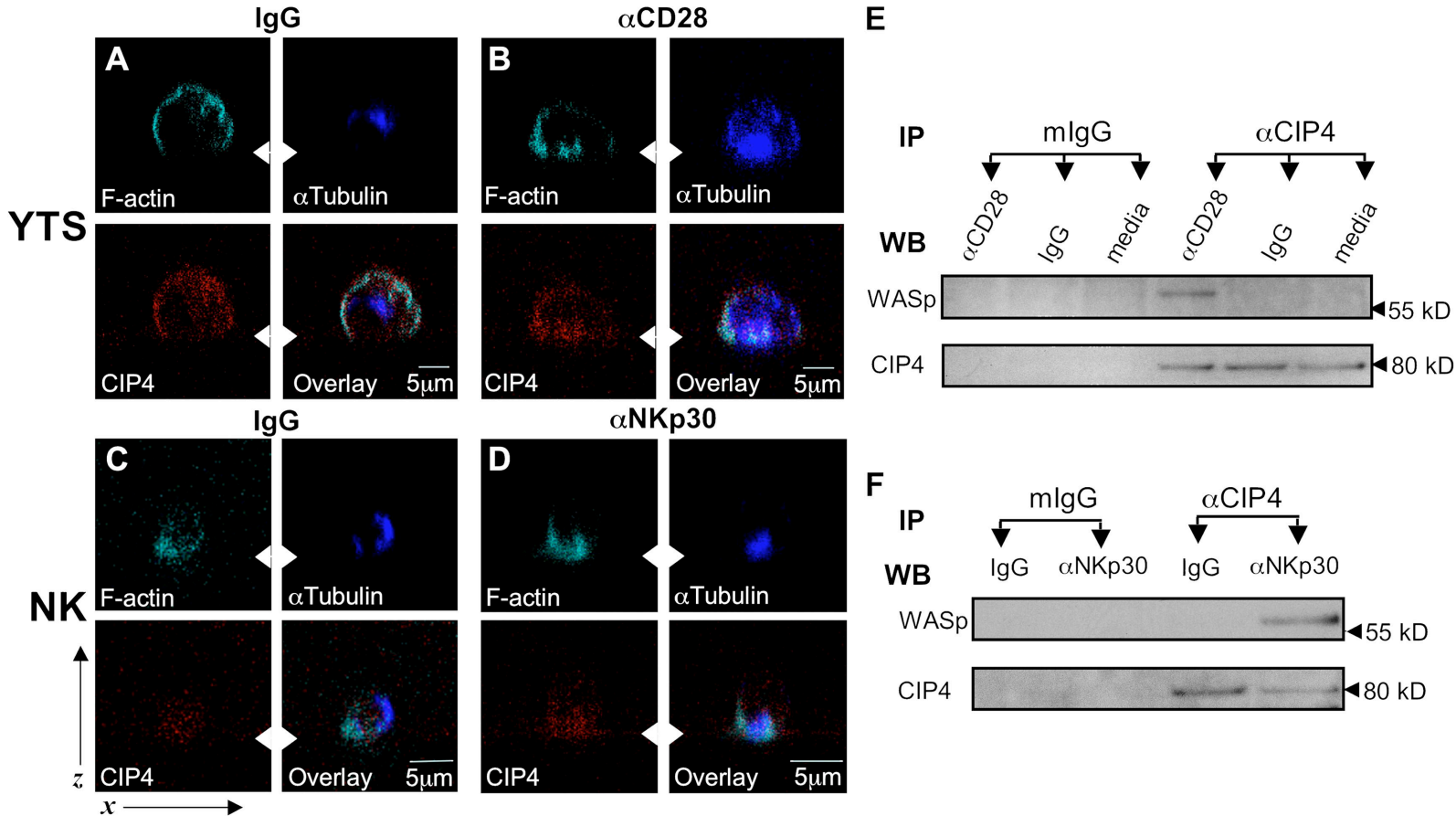

Figure 7. Alteration in association of endogenous CIP4 after NK cell activation. YTS cells were incubated on slides coated with IgG (A) or antiCD28 (B) and ex vivo NK cells on slides coated with lgG (C) or anti-NKp30 (D) for 30 min and evaluated via confocal microscopy in the $x-z$ plane. The arrowheads show the plane of the slide. Fluorescence demonstrating F-actin (cyan), $\alpha$-tubulin (blue), and CIP4 (red) is shown. (E) $10^{6}$ YTS cells were incubated in wells containing IgG, anti-CD28 mAb, or media for 30 min and then lysed. CIP4 was immunoprecipitated using mAb anti-CIP4 and probed for WASp and CIP4 by Western blotting. Immunoprecipitation with nonspecific mlgG was performed as a control. (F) $10^{7}$ ex vivo NK cells were lysed after a 30-min incubation in wells coated with IgG or anti-NKp30 mAb, and CIP4 was immunoprecipitated using mAb anti-CIP4. WASp and CIP4 were detected in immunoprecipitates by Western blotting. Immunoprecipitation with nonspecific mlgG was performed in parallel as a control. Blots represent at least three independent results. 
increased after YTS cells were exposed to anti-CD28 or ex vivo NK cells were exposed to anti-NKp30. Specifically, when activated cells were quantitatively analyzed and compared with values obtained from control-treated cells, the increase in colocalization between CIP4 and the MTOC was $27 \%$ in YTS cells and $24 \%$ in ex vivo NK cells ( $\geq 15$ cells over three separate experiments quantitatively evaluated for each condition). The increased colocalization between CIP4 and the MTOC after activating receptor signaling suggested that the CIP4 aggregates in the MTOC after activation. The colocalization between CIP4 and F-actin was also increased in cells on the activating antibody-coated surface $(15 \%$ in YTS cells and $21 \%$ in ex vivo NK cells). This suggested that CIP4 aggregated with the MTOC associates with the F-actin cortex after NK cell activation.

To biochemically discern an activation-induced association, CIP4 was immunoprecipitated from YTS cells that were exposed to plastic coated with IgG, media, or anti-CD28. The presence of WASp was evaluated in the immunoprecipitate by Western blotting (Fig. 7 E). Similar experiments were performed using ex vivo NK cells incubated on plastic surfaces coated with IgG or anti-NKp30 (Fig. 7 F). In either case, although CIP4 levels did not increase, there was an increased association between WASp and CIP4 after activation. Thus, after NK cell activation, CIP4 has an increased association with WASp and, therefore, has the potential to link actin branches in the cell cortex to the MTOC to serve a role in localizing the MTOC to the IS.

\section{CIP4 expression is required for NK cell cytotoxicity and MTOC polarization}

To demonstrate a role for endogenous CIP4 in MTOC polarization and cytotoxicity, small interfering RNA (siRNA) specific for CIP4 was used. siRNA was introduced into YTS cells via nucelofection, and siRNA directed against GAPDHor mock-transfected cells were used as controls. There were no differences found between GAPDH siRNA-transfected and mock-transfected cells for any of the parameters evaluated except for GAPDH expression (unpublished data). Introduction of siRNA specific for CIP4 reduced expression of CIP4 by $47.4 \pm 23.8 \%(\mathrm{P}<0.001)$ in YTS cells over nine independent experiments, as determined by Western blotting (Fig. 8 A). This reduction was specific to CIP4 siRNA, as GAPDH-specific siRNA did not reduce CIP4 expression. Similar results were obtained using a FACS analysis for CIP4 in siRNA nucleofected cells (unpublished data). Cytolytic activity was reduced by a mean of $42 \%$ across different effector/target cell ratios after CIP4 siRNA nucleofection when compared with GAPDH siRNA (Fig. 8 B). To determine if this was caused by an effect of reduced CIP4 expression on MTOC polarization to the IS, YTS cells nucleofected with either CIP4 or GAPDH siRNA were conjugated with KT86 target cells and evaluated via confocal microscopy. Although the accumulation of F-actin at the IS was comparable in both, there was a near $50 \%$ reduction in polarization of the tubulin-defined MTOC to the IS in cells nucleofected with CIP4 siRNA.
This demonstrated that IS formation had occurred in the presence of reduced CIP4 levels but that MTOC polarization was impaired. Therefore, the role of CIP4 in NK cell function is most likely in facilitating the localization of the MTOC to the IS, which is required for polarization of the lytic granules and cytotoxicity.

\section{Relation of CIP4 function to Cdc42 activation}

Because the activity of $\mathrm{Cdc} 42$ is required for the polarization of the MTOC to the IS (14), we wanted to determine the relation of CIP4 to Cdc42 activity. We assumed that CIP4 would function downstream of $\mathrm{Cdc} 42$, as interruption of $\mathrm{Cdc} 42$ activity interrupts both F-actin accumulation and MTOC polarization at the IS, whereas interference with CIP4 function only affected the latter. Two approaches were taken to determine if the requirement for CIP4 function followed

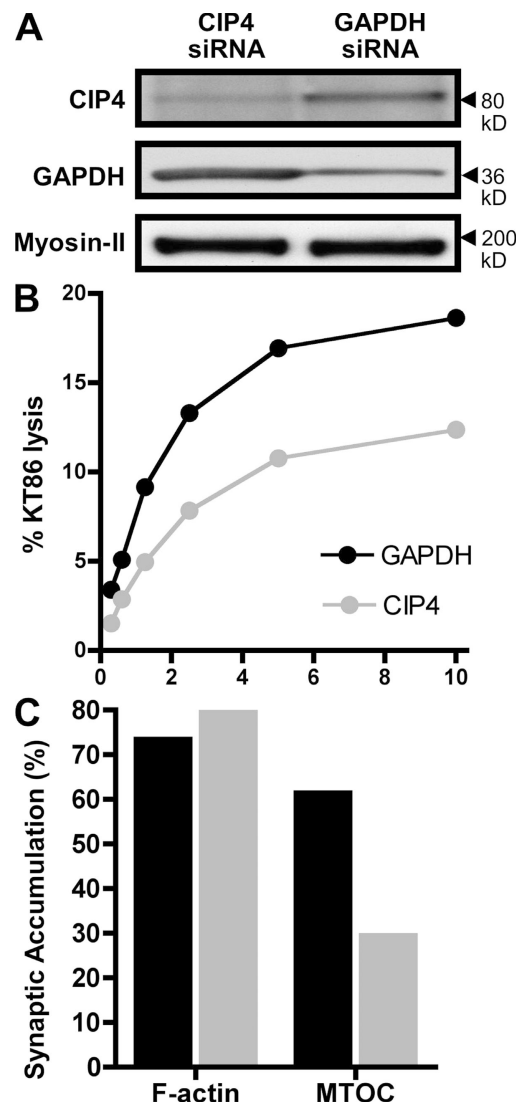

Figure 8. Requirement for CIP4 in NK cell cytotoxicity and MTOC polarization but not F-actin accumulation. (A) siRNA specific for CIP4 or GAPDH were introduced into YTS cells by nucleofection, and after $60 \mathrm{~h}$ were lysed and evaluated for the presence of CIP4 or GAPDH via Western blotting. Myosin-II was used as a loading control. (B) KT86 target-cell killing by YTS cells, $60 \mathrm{~h}$ after nucleofection of the YTS cells with either CIP4 or GAPDH siRNA. The $x$ axis represents the effector/target cell ratio. (C) F-actin accumulation at and MTOC polarization to the IS assessed by confocal microscopy in $\geq 50$ conjugates between YTS cells and KT86 cells $60 \mathrm{~h}$ after nucleofection of the YTS cells with either CIP4 (black bars) or GAPDH (gray bars) siRNA. All results represent at least three independent experiments. 
or preceded Cdc42 activation. The first was to nucleofect YTS cells with a previously described constitutively active Cdc42V12 construct $(15,25)$ and GAPDH or CIP4 siRNA. When these cells were conjugated with KT86 target cells to form a cytolytic IS, MTOC localization to the IS occurred normally in those that had received GAPDH, but not CIP4, siRNA (Fig. 9, A-L). The colocalization between the pericentrin and $\alpha$-tubulin-defined MTOC was not altered by CIP4 siRNA, suggesting that the integrity of the MTOC was not affected (Fig. 9 M). However, the polarization of the MTOC as measured by the mean distance of the pericentrin-defined MTOC to the IS was decreased after CIP4 siRNA nucleofection in the presence of constitutively active Cdc42V12 (Fig. 9 N). Concomitantly, the cytolytic activity of Cdc42V12 nucleofected cells that had received CIP4 siRNA compared with those that had received Cdc42V12 and GAPDH siRNA was reduced by $34 \pm 9 \%$ across effector/target cell ratios.
Collectively, these results demonstrated that CIP4 siRNA affects MTOC polarization and NK cell function even in the presence of active $\mathrm{Cdc} 42$.

As a second approach, active $\mathrm{Cdc} 42$ was isolated from anti-CD28-activated YTS cells (Fig. 9 O). In this case, active Cdc42 was present in activated YTS cells nucleofected with GFP, but there was more in YTS cells nucleofected with Cdc42V12, demonstrating the specificity of the assay. To evaluate the activation of $\mathrm{Cdc} 42$ in the presence of reduced CIP4, YTS cells were nucleofected with only CIP4 siRNA or GAPDH siRNA, activated, lysed, and active Cdc42 isolated. CIP4 siRNA did not reduce the amount of active Cdc42, suggesting that Cdc42 activation could occur independently of normal CIP4 expression. As a final consideration, YTS cells were compared with YTS cells overexpressing WT CIP4, which were inhibited in MTOC polarization and cytotoxicity. Again, the amount of active Cdc42 that could
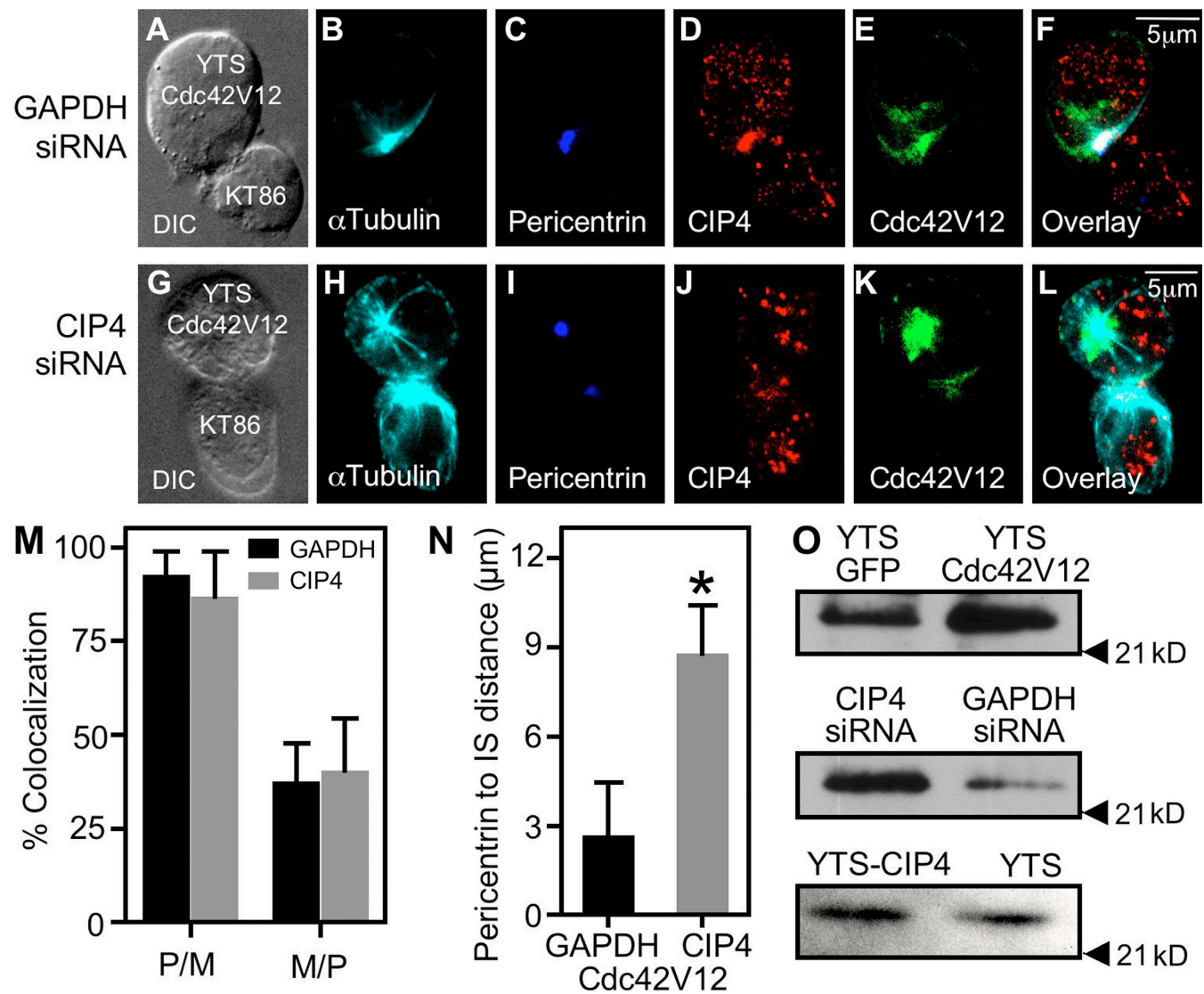

Figure 9. CIP4 function in MTOC polarization relative to Cdc42 activation. The cytolytic IS between a KT86 cell and a YTS cell nucleofected with constitutively active Cdc42V12-GFP and GAPDH siRNA (A-F) or CIP4 siRNA (G-L) are shown using DIC microscopy (A and G), as well as confocal microscopy showing fluorescence for $\alpha$-tubulin (B and H), pericentrin ( $C$ and I), CIP4 (D and J), Cdc42V12-GFP (E and K), and an overlay (F and L). (M) The mean percentage of the pericentrin-defined MTOC that colocalized with the tubulin-defined MTOC (P/M) and the percentage of the $\alpha$-tubulin-defined MTOC that colocalized with the pericentrin-defined MTOC (M/P) in YTS cells nucleofected with Cdc42V12-GFP and GAPDH siRNA (black bars) or Cdc42V12-GFP and CIP4 siRNA (gray bars), and conjugated with KT86 target cells, are shown. (N) Mean distance in micrometers of the pericentrin-defined MTOC to the IS in YTS cells nucleofected with Cdc42V12-GFP and GAPDH siRNA, or Cdc42V12-GFP and CIP4 siRNA. The increase in cells receiving CIP4 siRNA was significant. *, P $<0.01$. (0) Active Cdc42 pull down and Cdc42 Western blot from YTS cells activated with immobilized anti-CD28. Before activation, cells were nucleofected with GFP or Cdc42V12-GFP (top), or nucleofected with GAPDH siRNA or CIP4 siRNA (middle). Activated YTS cells were also compared with activated YTS cells overexpressing WT CIP4 (bottom). Error bars represent the SD. 
be isolated from activated YTS cells was not reduced by CIP4 overexpression. Thus, Cdc42 activation occurred in cells with interrupted CIP4 function, suggesting that CIP4 functions downstream of Cdc42 to induce MTOC polarization to the IS and cytotoxicity.

\section{DISCUSSION}

The cytolytic IS forms and functions in a series of stages. Some of these stages are linear in sequence and, thus, define key regulatory steps on which function of the IS depends. Previously, we found that F-actin and cell-surface receptor reorganization at the SMAC do not require microtubule function and precede the delivery of cytolytic granules to the IS (10). Lytic granule traffic, however, required both microtubule integrity and WASp-dependent F-actin function. Thus, these two events are sequential, suggesting the utility of potential signaling and structural linkages between them. Exact delivery of lytic granules is most likely a critical component of directed cytotoxicity and is consistent with work defining a specific cytolytic secretory domain at the edge of the central SMAC within the IS (26). This delivery of lytic granules to the IS has more recently been shown to be directed by the MTOC itself (11). Thus, localization of the MTOC is critical and may benefit from a specific connection between F-actin at the SMAC and microtubules. Because WASp is localized with F-actin at the pSMAC, including at the border of the secretory domain (16), a link between microtubules and WASp could serve a critical function in directing the MTOC and associated lytic granules to the secretory domain of the cytolytic IS. For these reasons, we investigated CIP4 for its potential to link WASp and tubulin as a critical regulator of the cytolytic immunological IS.

CIP4 was discovered because of its ability to bind to Cdc42 (17) and WASp (18) through two separate efforts using the yeast two-hybrid system. Overexpression of WASp and CIP4 in fibroblasts led to association of WASp with microtubules mediated by the FCH domain of CIP4 (18). It was subsequently appreciated that CIP4 deficient of its $\mathrm{FCH}$ or SH3 domains microinjected into macrophages could interfere with the formation of microtubule and WASp-dependent podosome formation (19). Collectively, these studies suggested the potential for CIP4 to provide relevant associations with both WASp and microtubules.

To determine if the CIP4 system was relevant to NK cell biology and potentially directed secretion, we initially identified that CIP4 was expressed in NK cells. CIP4 was diffusely present in cells that were not activated or in noncytolytic conjugates, as determined using confocal microscopy. After cell activation by either a target cell or NK cell activation receptor signaling, however, CIP4 accumulated with the MTOC (as defined either by $\alpha$-tubulin or pericentrin) at the IS (Figs. 2, 3, 6, 7, and 9; and Figs. S1, S2, and S4). This suggested that CIP4 functioned by joining the MTOC after cell activation. CIP4, however, could be coimmunoprecipitated with $\alpha$-tubulin even from resting NK cells in the absence of target cells (Fig. 5 A). This raised the possibility of a model in which CIP4 in resting cells is complexed with free tubulin, or with tubulin in individual microtubules, but is then focused to the MTOC during cell activation. Support for this model was consistent with CIP4 having reduced colocalization with the MTOC in resting NK cells or NK cells in noncytolytic conjugates, but was suggested by some $\alpha$-tubulin still being colocalized with CIP4. Moreover, the resolution of confocal microscopy is likely to underestimate the actual association between individual microtubules, or free tubulin, and CIP4. Also, as we have only evaluated $\alpha$-tubulin, there may be substantial associations between other forms of tubulin and CIP4, resulting in further underestimation.

Focusing CIP4 to the MTOC may have at least two functions. One would be in the actual polarization of the MTOC to the IS and the other in MTOC anchoring at the IS. The first of these two would require that CIP4 join the MTOC after cell activation but before MTOC polarization. A requirement for CIP4 in polarization of the MTOC is highlighted by the siRNA results and is further suggested by the overexpression studies in which polarization of the MTOC to the IS was decreased. Although MTOC polarization was reduced in cells overexpressing WT CIP4, this same result was also found in cells overexpressing CIP4 deleted of the $\mathrm{FCH}$ domain (Fig. $3 \mathrm{M}$ ). As this molecule is lacking its tubulin-interacting region, an alternative purpose to focusing CIP4 to the MTOC is suggested. Specifically, CIP4 may serve to anchor the MTOC at the appropriate location at the SMAC to allow directed delivery of the lytic granules. This anchoring function could be facilitated through the interaction of MTOC-associated CIP4 with WASp present in the F-actin cortex. Support for an anchoring function is provided by WASp-deficient NK cells, in which localization of the MTOC to the IS did not occur (Fig. 6). Alternatively, this result could be a feature of defective NK cell activation in the absence of WASp. In particular, ex vivo WAS NK cells demonstrate reduced adhesion to target cells (27), and those that have conjugated fail to cluster CD11a or CD11b at the IS (10). Thus, WASp-deficient NK cells may be receiving a weaker activation signal for MTOC polarization because of reduced integrin signaling. Our CIP4 overexpression studies, however, are also consistent with an MTOC-anchoring role for CIP4. Overexpressing CIP4 or CIP4 lacking its FCH domain would be capable of interacting with WASp without being associated with tubulin and, thus, could interfere with the interaction of tubulin-associated CIP4 with WASp. In this light, immunoprecipitation of CIP4 in cells overexpressing the WT construct yielded only slightly more tubulin than did CIP4 immunoprecipitated from parental cells (Fig. 5). Conversely, overexpression of the SH3-deleted construct of CIP4 may allow association with tubulin in the MTOC but prevent an anchoring function to WASp in the SMAC. Results obtained using CIP4 siRNA (Fig. 8) do not differentiate between these two models and only demonstrate a requirement for CIP4 in MTOC localization to the IS. As the MTOC is very dynamic in cytotoxic lymphocytes $(28,29)$, the ability to provide a strong anchor at the SMAC may be useful in 
maintaining the polarity of the MTOC. This, in turn is likely to be valuable in directing large numbers of lytic granules through the IS onto the target cell.

Given that CIP4 is infrequently found in the F-actin cell cortex in resting NK cells or in NK cells in noncytolytic conjugates, a specific mechanism for facilitating an interaction between CIP4 and WASp must be in place. Regulation could be mediated through the modification of CIP4, WASp, or both. Because CIP4 was associated with tubulin in resting cells (Fig. 5), the interaction with WASp may represent the critical step. Regulation of WASp has been extensively studied (for review see reference 30 ). WASp exists in an autoinhibited conformation in which its verprolin, cofilin, acidic domain and its GTPase binding domains are in contact, obscuring key elements required for F-actin branching (31). WASp autoinhibition is released after activation by the phosphorylation of key tyrosines. In unstimulated NK cells, where a majority of WASp would be expected to be autoinhibited, we found minimal WASp associated with CIP4 after CIP4 immunoprecipitation. As NK cell activation increased the association between CIP4 and WASp (Fig. 7, E and F), we propose that CIP4 preferentially interacts with activated WASp. The ability to detect WASp in the immunoprecipitates from cells overexpressing CIP4 could reflect the ability of a greatly increased number of CIP4 molecules to interact with the relatively small amount of activated WASp that exists in resting cells. Alternatively, CIP4 overexpression could influence the activation state of WASp specifically. An interaction between CIP4 and activated WASp, however, would present a useful means for specifically anchoring the MTOC at the SMAC, where WASp is functioning to rearrange F-actin. Similarly, it would present a useful means of releasing the MTOC once signaling is terminated and WASp reverts to its autoinhibited state. Although further study of MTOC dynamics will be required to specifically prove this hypothesis, in preliminary experiments we did find phosphorylated WASp associated with CIP4 after physiologic NK cell activation (unpublished data). As WASp is expressed only in hematopoietic cells, this would represent a hematopoietic cell-specific mechanism for positioning and/or anchoring the MTOC. CIP4 does interact with the more ubiquitously expressed WASp homologue neural WASp $(32,33)$ and, thus, can serve a more general role in MTOC positioning. However, because neural WASp does not completely compensate for WASp deficiency in NK cells $(16,27)$, the interaction between WASp and CIP4 may have a specific role in controlling MTOC positioning in the immune system.

Not all lytic granules, however, require the MTOC and microtubule function for activation-induced polarization to the IS. Some lytic granules are "predocked" with the NK cell membrane (34) and are most likely poised for rapid release onto target cells without the need for substantial cytoskeletal rearrangement. This may in part explain why the CIP4-overexpressing cells, or cells nucleofected with CIP4 siRNA, still mediate some cytotoxicity even after having reduced MTOC polarization (Fig. $4 \mathrm{D}$ and Fig. 8 B). Alternatively, cytotoxicity in these cells was mediated only by the subset in which MTOC polarization occurred. It is more likely that CIP4 as well as MTOC polarization and anchoring are likely to only be required for a portion of NK cell cytotoxicity. Specifically, they would be required for that which depends on granule delivery from elsewhere in the cell to the IS. For this reason, we also considered it important to use ex vivo NK cells that had not been cultured or otherwise prestimulated in the experiments. Activation signals, such as those commonly provided when culturing NK cells, could increase the percentage of lytic granules that are predocked at the cell membrane and reduce reliance on the microtubule-directed transport of granules. Alternatively, NK cell activation signals may induce different mechanisms of enabling MTOC anchoring at the IS. In this light, NK cell activation with IL-2 did not increase the cellular levels of CIP4 but did increase MTOC polarization (unpublished data). Even without activation, however, any linkage between the MTOC and F-actin in the SMAC may be redundant. As evidence, the residual MTOC polarization and cytotoxicity in the CIP4-overexpressing and knockdown cells may reflect alternative mechanisms for MTOC anchoring at the IS. A candidate alternate protein is LIS1, which facilitates the attachment of microtubules to the cell cortex in Dictyostelium through its ability to associate with dynein and Rac1 (35). In contrast to CIP4, however, LIS1 is inherently associated with the centrosome and is also involved in actin reorganization. Our earlier experiments suggest the existence of a linking protein that is not required for actin dynamics, because microtubule integrity (as disrupted by colchicine treatment) is not required for actin accumulation at the SMAC (10).

Finally, as CIP4 can also interact with Cdc42 (17) and Cdc42 is required for MTOC reorientation to the IS (14), the function of CIP4 could have been through facilitating Cdc42 activity. Because interfering with Cdc42 activity reduces F-actin reorganization at the IS (14) and lytic granule polarization to the IS occurs after F-actin has accumulated (10), we focused on a protein that would block MTOC polarization without interfering with $\mathrm{F}$-actin accumulation at the SMAC. CIP4 functions downstream of $\mathrm{Cdc} 42$, as the effect of reducing CIP4 in blocking MTOC reorientation was still apparent in the presence of constitutively active Cdc 42 (Fig. 9). Furthermore, after NK cell activation, active Cdc 42 levels were unaffected by CIP4 siRNA or CIP4 overexpression (Fig. 9). Thus, it is likely that Cdc42 function represents another early requirement in facilitating the signals leading to MTOC reorientation more akin to WASp, which was required for both F-actin accumulation and MTOC polarization (Fig. 6). It is possible, however, that CIP4 in the MTOC may use Cdc42 as an additional anchor to facilitate MTOC positioning.

As directing lytic granules to the IS is a required component of the specific lysis of target cells, the ability to direct and anchor the MTOC at the IS can be an essential step in effective lytic function. Polarizing and anchoring the MTOC 
can allow for the delivery of substantial numbers of lytic granules to the IS and enable maximal exocytosis of lytic granules. CIP4 is a protein that participates in this process first through being focused to the MTOC and then by interacting with WASp. CIP4 has the functional capacity to link actin and microtubule networks in NK cells downstream of $\mathrm{Cdc} 42$, and its appropriate expression is required for polarization of the MTOC to the cytolytic IS. CIP4 presents an important paradigm in NK cells that may apply to other immune cells, by which immunological activity can be enabled through functional molecular linkage between the SMAC and MTOC.

\section{MATERIALS AND METHODS}

NK cell preparation, NK cell lines, and cellular evaluation. Leukocyte-enriched blood was obtained from volunteer donors and was used to prepare ex vivo NK cells by negative depletion using the human NK cell isolation kit II (Miltenyi Biotec). Ex vivo NK cells from a patient with a G252A point mutation in WASP resulting in undetectable WASp expression (16) were prepared from peripheral blood. NK cell preparations contained $>95 \% \mathrm{CD}^{2} 6^{+} / \mathrm{CD}^{-}$cells with $<1 \% \mathrm{CD}^{+}$cells, as determined by FACS using fluorophore-conjugated $\mathrm{mAbs}$ (BD Biosciences). All human samples were obtained after informed patient or parental consent and were used with approval of the institutional internal review board for the protection of human subjects of the Children's Hospital of Philadelphia or the Children's Hospital Boston. The immortalized NK cell lines YTS, YTS expressing the mouse ecotropic receptor (YTSeco), YTS GFP expressing GFP, YTS CD2GFP expressing a CD2-GFP fusion protein, and NK92, as well as the MHC class I-negative K562 erythroleukemia target cell line, have all been previously used and described $(10,36)$. GFP-expressing K562 cells (K562-GFP) were created by transfection of K562 cells with a GFP expression vector (CLONTECH Laboratories, Inc.) containing a neomycin resistance gene and GFP maintained via neomycin selection. K562 cells stably expressing CD86 (KT86 cells) were created with CD86 cloned from cDNA (prepared from matured dendritic cells), which was then cloned into lentiviral vectors, as previously described (37). Lentivirus-transduced K562 cells were sorted for high CD86 expression, and the KT86 line was derived from a single-cell clone. NK cell cytotoxicity against KT86 target cells was evaluated by a 4-h ${ }^{51} \mathrm{Cr}$ release assay, as previously described (16).

Molecular cloning and analysis. RNA was isolated using TRIzol reagent (Invitrogen) precipitated with isopropanol and washed twice with $75 \%$ ethanol. Equal amounts of RNA were subjected to RT-PCR for CIP4 using the Advantage RT-for-PCR kit (CLONTECH Laboratories, Inc.) and the following primers: 5'-TGGTGCCCATAATAGCCAA-3' and $5^{\prime}-$ TTTCAGCCGTTCAATGTTGC- $3^{\prime}$ to amplify the middle $500 \mathrm{nt}$ of CIP4. Products were visualized on a $1 \%$ agarose gel and sequenced at the nucleic acid core facility of the Children's Hospital of Philadelphia. Other overlapping primer sets were used to confirm the consensus of CIP4 in NK cells (available from GenBank/EMBL/DDBJ under accession no. AJ000414).

Full-length CIP4 (encoding aa 1-545), FCH domain-deleted CIP4 (encoding aa 118-545) and SH3 domain-deleted CIP4 (encoding aa 1-417) were provided by D.M. Stewart and D.L. Nelson (National Institutes of Health, Bethesda, MD). StuI and NotI restriction sites were added to these constructs via overhanging PCR amplification to allow cloning into pTOPO zero blunt (Invitrogen) and, subsequently, the bicistronic retroviral expression vector pMX-IRES-GFP (38). Vectors were packaged and used to transduce YTSeco cells, as previously described (10), and transductants expressing consistent levels of transgene were sorted by FACS (FACSVantage; BD Biosciences). Full-length CIP4 was also detected by intracytoplasmic FACS using the Cytofix/Cytoperm reagents (BD Biosciences), where specified in the figures, and the anti-CIP4 mAb clone 21 or IgG clone MOPC21 (BD Biosciences) as a primary antibody and Alexa Fluor 647-conjugated goat anti-mouse (Invitrogen) as a secondary reagent.
Immunoprecipitation and Western blotting. Cells were lysed in icecold lysis buffer (50 mM Tris-Cl [pH 7.5], $100 \mathrm{mM} \mathrm{NaCl,} 15 \mathrm{mM}$ EDTA, $0.1 \%$ Triton X-100) and protease inhibitor cocktail (Roche). For Western blotting of total lysate, $10 \mu \mathrm{g}$ of protein per sample was separated on a $4-12 \%$ Bis-Tris density gradient gel (Invitrogen) and transferred to polyvinylidene fluoride membranes, which after blocking with $3 \% \mathrm{BSA}$ and $140 \mathrm{mM} \mathrm{NaCl}$ in Tris-buffered saline (TBS) were incubated with mouse anti-CIP4 $\mathrm{mAb}$ clone 21 (BD Biosciences). Bound antibody was detected with peroxidaseconjugated light chain-specific goat anti-mouse IgG (Jackson ImmunoResearch Laboratories) and an enhanced chemiluminescence detection system (GE Healthcare). Arrowheads shown in Western blot images denote the location of molecular mass markers. Membranes were stripped in $0.2 \mathrm{M}$ glycine ( $\mathrm{pH} 2.5$ ), $0.05 \%$ Tween 20 , and $140 \mathrm{mM} \mathrm{NaCl}$ in TBS at $50^{\circ} \mathrm{C}$ for 30 min, blocked with 3\% BSA, and reprobed with rabbit anti-WASp pAb (U.S. Biologicals) or rabbit antitubulin pAb (U.S. Biologicals), followed by peroxidase-conjugated light chain-specific goat anti-rabbit IgG (Jackson ImmunoResearch Laboratories). In gene silencing experiments, GAPDH was detected using mouse anti-GAPDH clone ID4 (Imgenex). For immunoprecipitation, lysates were normalized for either protein content or cell number and precleared with $10 \mu \mathrm{g} / \mathrm{ml}$ of mouse mAb clone MOPC21 (BD Biosciences) and protein $G$ agarose beads (Invitrogen). The supernatant was incubated with $20 \mu \mathrm{g} / \mathrm{ml}$ anti-CIP4 $\mathrm{mAb}$ or nonspecific $\mathrm{mAb}$ IgG MOPC21, followed by protein $\mathrm{G}$ agarose beads, and the immunoprecipitate was separated on density gradient gels, followed by Western blotting. For preactivation of cells through CD28 or NKp30, untreated polystyrene sixwell plates (Nunc) were coated with $5 \mu \mathrm{g} / \mathrm{ml}$ of $\mathrm{mAb}$ anti-CD28 clone 9.3 (a gift of J. Riley, University of Pennsylvania School of Medicine, Philadelphia, PA) or anti-NKp30 clone Z25 (Beckman Coulter), and were washed and incubated with $10^{6}$ YTS or $10^{7}$ ex vivo NK cells for $30 \mathrm{~min}$ at $37^{\circ} \mathrm{C}$. Wells coated with mAb MOPC21 were used as a control.

Microtubule binding protein spin-down assay. The assay was performed using a commercially available reagent set according to the manufacturer's recommendations (Cytoskeleton, Inc.). In brief, microtubules were generated from purified tubulin and stabilized with $200 \mu \mathrm{M}$ Taxol. YTS cells were lysed in $20 \mathrm{mM}$ of ice-cold Hepes (pH 7), $2 \mathrm{mM} \mathrm{EGTA}$, and $0.5 \%$ Triton X-100 and with protease inhibitor cocktail for $1.5 \mathrm{~h}$, and were cleared of debris by centrifugation at $9,300 \mathrm{~g}$. The cleared lysate was then incubated for $30 \mathrm{~min}$ at room temperature in the presence or absence of $10 \mathrm{nM}$ of stabilized microtubules. Microtubules and associated proteins were precipitated by centrifugation at $100,000 \mathrm{~g}$ through $80 \mathrm{mM}$ Pipes ( $\mathrm{pH} 7$ ), $1 \mathrm{mM} \mathrm{MgCl}_{2}, 1 \mathrm{mM}$ EGTA, and 50\% glycerol with Taxol. The precipitate and supernatant were evaluated for the presence of CIP4 and tubulin by Western blotting.

Confocal microscopy and image analysis. Conjugates between NK cells and target cells at a 2:1 ratio were formed in suspension for $15 \mathrm{~min}$ and adhered to poly-L-lysine-coated glass slides (Polyprep; Sigma-Aldrich) for $15 \mathrm{~min}$, all at $37^{\circ} \mathrm{C}$, as previously described (10). In experiments evaluating NK cell activation without target cells, poly-L-lysine-coated glass slides were coated with IgG MOPC21 as a control, or anti-CD28, or anti-NKp30 by overnight incubation with $5 \mu \mathrm{g} / \mathrm{ml}$ of antibody in PBS. Slides were washed, and NK cells were incubated on the slide in media for $30 \mathrm{~min}$ at $37^{\circ} \mathrm{C}$, after which nonadherent cells were rinsed. In either case, cells adhering to the slide were fixed and permeabilized with $4 \%$ formaldehyde, $0.1 \%$ saponin, and $0.1 \%$ Triton X-100 in PBS for $15 \mathrm{~min}$, rinsed, and incubated for $1 \mathrm{~h}$ with anti-CIP4 $\mathrm{mAb}$ or nonspecific IgG clone MOPC21 (as a control). Slides were rinsed, incubated for $1 \mathrm{~h}$ with Alexa Fluor 647-conjugated highly cross-adsorbed goat anti-mouse (Invitrogen), rinsed again, and incubated with $10 \%$ heat-inactivated mouse serum (Sigma-Aldrich) for $30 \mathrm{~min}$ to block nonspecific binding. Slides were rinsed and incubated with biotinylated anti- $\alpha$-tubulin $\mathrm{mAb}$ (Invitrogen) or biotinylated mouse IgG control (BD Biosciences) for $1 \mathrm{~h}$, followed by additional rinsing and incubation with streptavidin-conjugated Pacific blue 405 (Invitrogen) and Alexa Fluor 568conjugated phalloidin (Invitrogen). Slides were rinsed and covered with 
0.15-mm glass coverslips (VWR Scientific) using Prolong Antifade reagent (Invitrogen) or Vectashield with DAPI . Other antibodies used for microscopy, where specified in the figures, include $\mathrm{pAb}$ rabbit anti- $\alpha$-tubulin (Abcam), $\mathrm{pAb}$ antipericentrin (Abcam), Alexa Fluor 568-conjugated anti-rabbit IgG (Invitrogen), FITC anti-rabbit IgG (Jackson ImmunoResearch Laboratories), FITC anti-mouse IgG (Jackson ImmunoResearch Laboratories), and $\mathrm{mAb}$ FITC antiperforin (Becton Dickenson). All antibodies were used in the range of $1-20 \mu \mathrm{g} / \mathrm{ml}$ and were diluted in saponin-containing buffer. For each experiment, cells stained with individual fluorophores and controls for individual fluorophores were included as controls to allow for proper spectral separation and/or adjustment of the microscope.

Cell conjugates were visualized by using either a laser scanning confocal microscope (DMIRE2; Leica) or a spinning-disc confocal microscope (IX81 DSU; Olympus) via scanning through the $\mathrm{x}-\mathrm{y}$ plane. Detection settings were adjusted so that a control-stained sample was uniformly negative and experimentally stained samples were not saturating or bleeding into other channels. The effector and target cells in the conjugate were confirmed either by size (for ex vivo NK cells) or by the presence or absence of GFP expression. The area of accumulation of fluorescent molecules in the NK cell was examined using the threshold of $\geq 40 \%$ of unaccumulated fluorescence intensity for evaluations of CD2 or F-actin (a number based on previously applied assumptions) $(10,28)$. Accumulation of CD2 or F-actin at the IS consisted of an increased area of accumulated $\mathrm{CD} 2$ or F-actin at the IS relative to other cell-surface or cortical regions. Accumulated areas of CIP4, $\alpha$-tubulin, and pericentrin were identified using the same threshold approach but were often not found at the IS or in the cell cortex. To determine the area occupied by an accumulated fluorescence intensity and colocalization between areas of different fluorescence, images were analyzed using the Volocity software package classification module (Improvision). The area occupied by each accumulated fluorophore only in the effector cell was measured. Areas containing two accumulated fluorophores were also measured and compared with the area occupied by the single fluorophore to calculate the percentage colocalization. The MTOC was defined either as the accumulated area of $\alpha$-tubulin using a pixel-size threshold, to eliminate measurement of isolated microtubules, or as the accumulated area of the centrosomal protein pericentrin. As an alternative approach to evaluating colocalization with the MTOC, fluorescence intensities of the individual pixels that were over background levels in a $5-\mu \mathrm{m}^{2}$ region enclosing the MTOC were measured in some experiments, as noted in the figures. In experiments evaluating NK cell activation by a coated glass surface, scanning was performed using the Leica microscope scanning in the $\mathrm{x}-\mathrm{z}$ plane to completely visualize the interface with the glass, and analysis was performed as for the conjugates. Polarization of the MTOC to the IS was defined as either there being at least some degree of colocalization of the $\alpha$-tubulin-defined fluorescent region with cell-surface $\mathrm{CD} 2$, or cortical F-actin at the contact site with the target cell, or having some contact with the glass surface in $\mathrm{x}-\mathrm{z}$ experiments. The shortest distance from the centroid of the accumulated fluorescence and the cell membrane (identified via differential interference contrast [DIC] microscopy) or the edge of the nucleus (identified via DAPI staining) was also measured using Volocity in some experiments to further define and evaluate polarization. In experiments using siRNA-transfected YTS cells, perforin was visualized using FITC-conjugated mouse anti-human perforin clone 27-35 (BD Biosciences) to identify the effector cell in conjugates formed between YTS and KT86 cells.

RNA interference and nucleofection. siRNA containing oligonucleotides with specificity for either CIP4 (Dharmacon siGenome SmartPool) or GAPDH (Dharmacon siGenome Duplex) control were introduced into YTS cells using a nucleofector (program O-017 with reagent R; Amaxa). Titration experiments using CIP4 Western blotting and CIP4 flow cytometry were performed to determine the optimal concentration of CIP4 siRNA, the optimal number of cells per cuvette, and the optimal incubation time after nucleofection. $2.5 \mu \mathrm{M}$ of siRNA, $2-3 \times 10^{6}$ cells per cuvette, and $60 \mathrm{~h}$ of incubation after nucleofection were ultimately chosen. Although GAPDH expression could be suppressed using as little as $100 \mathrm{nM}$ siRNA, the use of higher concentrations to control for the CIP4 siRNA did not have any nonspecific effect. Constitutively active Cdc42V12 (39), as a GFP-Cdc42V12 fusion construct (provided in expression vector by M. Chou, University of Pennsylvania, Philadelphia, PA) (25), was nucleofected into YTS cells using $2 \mu \mathrm{g}$ of plasmid, along with either CIP4 or GAPDH siRNA, and incubated for $48 \mathrm{~h}$ before use in experiments. $50-60 \%$ of cells were GFP positive after incubation. Localization of Cdc42 V12 was determined by microscopy, and increased active $\mathrm{Cdc} 42$ was confirmed by an active $\mathrm{Cdc} 42$ pull-down assay.

Statistical analysis. All graphs denote mean values, except where noted, and error bars represent the SD. Comparisons of groups of data were performed using the two-tailed Student's $t$ test, and statistical significance is shown in figures $(*, P<0.01$, unless otherwise noted).

Active Cdc42 pull-down assay. Active Cdc42 was isolated using the EZdetect Cdc42 activation kit (Pierce Chemical Co.), according to the manufacturer's protocol. In brief, clarified YTS cell lysates prepared from equal numbers of cells were treated with $0.1 \mathrm{mM} \mathrm{GTP} \gamma \mathrm{S}$ in the presence of $10 \mu \mathrm{M}$ EDTA ( $\mathrm{pH} 8$ ) at $30^{\circ} \mathrm{C}$ for 15 min to activate $\mathrm{Cdc} 42$, after which the nucleotide exchange reaction was terminated by adding $\mathrm{MgCl}_{2}$ and placing samples on ice. Treated lysates were incubated with GST-Pak1-PBD in the presence of SwellGel Immobilized Glutathione (Pierce Chemical Co.) at $4^{\circ} \mathrm{C}$ for $1 \mathrm{~h}$. After incubation, the mixture was centrifuged at $2,000 \mathrm{~g}$, and the precipitate was washed three times before boiling to isolate bound active Cdc42. Cdc42 was identified in the precipitate by Western blotting using $\mathrm{mAb}$ anti-Cdc42.

Online supplemental material. Fig. S1 demonstrates the location of the pericentrin-defined MTOC and CIP4 in cytolytic and noncytolytic conjugates in YTS cells. Fig. S2 contains an analysis of colocalization in the $5-\mu \mathrm{m}^{2}$ region containing the MTOC and an enlargement of detail within that region in cytolytic and noncytolytic conjugates in YTS cells. Fig. S3 depicts perforin localization, along with the MTOC, and cytolytic IS in YTS cells overexpressing WT CIP4 or truncated CIP4. Fig. S4 demonstrates CIP4 localization relative to the pericentrin-defined MTOC and IS in ex vivo NK cells. Online supplemental material is available at http://www.jem .org/cgi/content/full/jem.20061893/DC1.

This work was supported by National Institutes of Health grants Al055602 and Al067946, and a faculty development award from the Education Research Trust of the American Academy of Allergy and Immunology.

The authors have no conflicting financial interests.

Submitted: 5 September 2006

Accepted: 2 August 2007

\section{REFERENCES}

1. Davis, D.M., and M.L. Dustin. 2004. What is the importance of the immunological synapse? Trends Immunol. 25:323-327.

2. Poo, W.J., L. Conrad, and C.A. Janeway Jr. 1988. Receptor-directed focusing of lymphokine release by helper T cells. Nature. 332:378-380.

3. Kupfer, A., T.R. Mosmann, and H. Kupfer. 1991. Polarized expression of cytokines in cell conjugates of helper T cells and splenic B cells. Proc. Natl. Acad. Sci. USA. 88:775-779.

4. Kupfer, A., G. Dennert, and S.J. Singer. 1983. Polarization of the Golgi apparatus and the microtubule-organizing center within cloned natural killer cells bound to their targets. Proc. Natl. Acad. Sci. USA. 80:7224-7228.

5. Peters, P.J., J. Borst, V. Oorschot, M. Fukuda, O. Krahenbuhl, J. Tschopp, J.W. Slot, and H.J. Geuze. 1991. Cytotoxic T lymphocyte granules are secretory lysosomes, containing both perforin and granzymes. J. Exp. Med. 173:1099-1109.

6. Menasche, G., E. Pastural, J. Feldmann, S. Certain, F. Ersoy, S. Dupuis, N. Wulffraat, D. Bianchi, A. Fischer, F. Le Deist, and G. de Saint Basile. 2000. Mutations in RAB27A cause Griscelli syndrome associated with haemophagocytic syndrome. Nat. Genet. 25:173-176. 
7. Feldmann, J., I. Callebaut, G. Raposo, S. Certain, D. Bacq, C. Dumont, N. Lambert, M. Ouachee-Chardin, G. Chedeville, H. Tamary, et al. 2003. Munc13-4 is essential for cytolytic granules fusion and is mutated in a form of familial hemophagocytic lymphohistiocytosis (FHL3). Cell. 115:461-473.

8. zur Stadt, U., S. Schmidt, B. Kasper, K. Beutel, A.S. Diler, J.I. Henter, H. Kabisch, R. Schneppenheim, P. Nurnberg, G. Janka, and H.C. Hennies. 2005. Linkage of familial hemophagocytic lymphohistiocytosis (FHL) type-4 to chromosome 6q24 and identification of mutations in syntaxin 11. Hum. Mol. Genet. 14:827-834.

9. Burkhardt, J.K., J.M. McIlvain Jr., M.P. Sheetz, and Y. Argon. 1993. Lytic granules from cytotoxic $\mathrm{T}$ cells exhibit kinesin-dependent motility on microtubules in vitro. J. Cell Sci. 104:151-162.

10. Orange, J.S., K.E. Harris, M.M. Andzelm, M.M. Valter, R.S. Geha, and J.L. Strominger. 2003. The mature activating natural killer cell immunologic synapse is formed in distinct stages. Proc. Natl. Acad. Sci. USA. 100:14151-14156.

11. Stinchcombe, J.C., E. Majorovits, G. Bossi, S. Fuller, and G.M. Griffiths. 2006. Centrosome polarization delivers secretory granules to the immunological synapse. Nature. 443:462-465.

12. Kopcow, H.D., D.S. Allan, X. Chen, B. Rybalov, M.M. Andzelm, B. Ge, and J.L. Strominger. 2005. Human decidual NK cells form immature activating synapses and are not cytotoxic. Proc. Natl. Acad. Sci. USA. 102:15563-15568.

13. Chen, X., D.S. Allan, K. Krzewski, B. Ge, H. Kopcow, and J.L. Strominger. 2006. CD28-stimulated ERK2 phosphorylation is required for polarization of the microtubule organizing center and granules in YTS NK cells. Proc. Natl. Acad. Sci. USA. 103:10346-10351.

14. Stowers, L., D. Yelon, L.J. Berg, and J. Chant. 1995. Regulation of the polarization of $\mathrm{T}$ cells toward antigen-presenting cells by Ras-related GTPase CDC42. Proc. Natl. Acad. Sci. USA. 92:5027-5031.

15. Tskvitaria-Fuller, I., A. Seth, N. Mistry, H. Gu, M.K. Rosen, and C. Wulfing. 2006. Specific patterns of Cdc42 activity are related to distinct elements of T cell polarization. J. Immunol. 177:1708-1720.

16. Orange, J.S., N. Ramesh, E. Remold-O’Donnell, Y. Sasahara, L. Koopman, M. Byrne, F.A. Bonilla, F.S. Rosen, R.S. Geha, and J.L. Strominger. 2002. Wiskott-Aldrich syndrome protein is required for NK cell cytotoxicity and colocalizes with actin to NK cell-activating immunologic synapses. Proc. Natl. Acad. Sci. USA. 99:11351-11356.

17. Aspenstrom, P. 1997. A Cdc42 target protein with homology to the non-kinase domain of FER has a potential role in regulating the actin cytoskeleton. Curr. Biol. 7:479-487.

18. Tian, L., D.L. Nelson, and D.M. Stewart. 2000. Cdc42-interacting protein 4 mediates binding of the Wiskott-Aldrich syndrome protein to microtubules. J. Biol. Chem. 275:7854-7861.

19. Linder, S., K. Hufner, U. Wintergerst, and M. Aepfelbacher. 2000. Microtubule-dependent formation of podosomal adhesion structures in primary human macrophages. J. Cell Sci. 113:4165-4176.

20. Lu, Y., R. Rodriguez, J. Bjorndahl, C.A. Phillips, and J.M. Trevillyan. 1996. CD28-dependent killing by human YT cells requires phosphatidylinositol 3-kinase activation. Eur. J. Immunol. 26:1278-1284.

21. Teng, J.M., X.R. Liu, G.B. Mills, and B. Dupont. 1996. CD28-mediated cytotoxicity by the human leukemic NK cell line YT involves tyrosine phosphorylation, activation of phosphatidylinositol 3-kinase, and protein kinase C. J. Immunol. 156:3222-3232.

22. Cohen, G.B., R.T. Gandhi, D.M. Davis, O. Mandelboim, B.K. Chen, J.L. Strominger, and D. Baltimore. 1999. The selective downregulation of class I major histocompatibility complex proteins by HIV-1 protects HIV-infected cells from NK cells. Immunity. 10:661-671.

23. Pende, D., S. Parolini, A. Pessino, S. Sivori, R. Augugliaro, L. Morelli, E. Marcenaro, L. Accame, A. Malaspina, R. Biassoni, et al. 1999. Identifi- cation and molecular characterization of NKp30, a novel triggering receptor involved in natural cytotoxicity mediated by human natural killer cells. J. Exp. Med. 190:1505-1516.

24. Bryceson, Y.T., M.E. March, D.F. Barber, H.G. Ljunggren, and E.O. Long. 2005. Cytolytic granule polarization and degranulation controlled by different receptors in resting NK cells. J. Exp. Med. 202: 1001-1012

25. Luna, A., O.B. Matas, J.A. Martinez-Menarguez, E. Mato, J.M. Duran, J. Ballesta, M. Way, and G. Egea. 2002. Regulation of protein transport from the Golgi complex to the endoplasmic reticulum by CDC42 and N-WASP. Mol. Biol. Cell. 13:866-879.

26. Stinchcombe, J.C., G. Bossi, S. Booth, and G.M. Griffiths. 2001. The immunological synapse of CTL contains a secretory domain and membrane bridges. Immunity. 15:751-761.

27. Gismondi, A., L. Cifaldi, C. Mazza, S. Giliani, S. Parolini, S. Morrone, J. Jacobelli, E. Bandiera, L. Notarangelo, and A. Santoni. 2004. Impaired natural and CD16-mediated NK cell cytotoxicity in patients with WAS and XLT: ability of IL-2 to correct NK cell functional defect. Blood. 104:436-443.

28. Wulfing, C., B. Purtic, J. Klem, and J.D. Schatzle. 2003. Stepwise cytoskeletal polarization as a series of checkpoints in innate but not adaptive cytolytic killing. Proc. Natl. Acad. Sci. USA. 100:7767-7772.

29. Kuhn, J.R., and M. Poenie. 2002. Dynamic polarization of the microtubule cytoskeleton during CTL-mediated killing. Immunity. 16: 111-121.

30. Orange, J.S., K.D. Stone, S.E. Turvey, and K. Krzewski. 2004. Biomedicine and disease: the Wiskott-Aldrich syndrome. Cell. Mol. Life Sci. 61:2361-2385.

31. Kim, A.S., L.T. Kakalis, N. Abdul-Manan, G.A. Liu, and M.K. Rosen. 2000. Autoinhibition and activation mechanisms of the Wiskott-Aldrich syndrome protein. Nature. 404:151-158.

32. Tsujita, K., S. Suetsugu, N. Sasaki, M. Furutani, T. Oikawa, and T. Takenawa. 2006. Coordination between the actin cytoskeleton and membrane deformation by a novel membrane tubulation domain of $\mathrm{PCH}$ proteins is involved in endocytosis. J. Cell Biol. 172:269-279.

33. Itoh, T., K.S. Erdmann, A. Roux, B. Habermann, H. Werner, and P. De Camilli. 2005. Dynamin and the actin cytoskeleton cooperatively regulate plasma membrane invagination by $\mathrm{BAR}$ and F-BAR proteins. Dev. Cell. 9:791-804.

34. Liu, D., L. Xu, F. Yang, D. Li, F. Gong, and T. Xu. 2005. Rapid biogenesis and sensitization of secretory lysosomes in NK cells mediated by target-cell recognition. Proc. Natl. Acad. Sci. USA. 102:123-127.

35. Rehberg, M., J. Kleylein-Sohn, J. Faix, T.H. Ho, I. Schulz, and R. Graf. 2005. Dictyostelium LIS1 is a centrosomal protein required for microtubule/cell cortex interactions, nucleus/centrosome linkage, and actin dynamics. Mol. Biol. Cell. 16:2759-2771.

36. Li, Y., T. Zhang, C. Ho, J.S. Orange, S.D. Douglas, and W.Z. Ho. 2004. Natural killer cells inhibit hepatitis C virus expression. J. Leukoc. Biol. 76:1171-1179.

37. Parry, R.V., C.A. Rumbley, L.H. Vandenberghe, C.H. June, and J.L. Riley. 2003. CD28 and inducible costimulatory protein Src homology 2 binding domains show distinct regulation of phosphatidylinositol 3kinase, $\mathrm{Bcl}-\mathrm{xL}$, and IL-2 expression in primary human CD4 T lymphocytes. J. Immunol. 171:166-174.

38. Liu, X., S.N. Constantinescu, Y. Sun, J.S. Bogan, D. Hirsch, R.A. Weinberg, and H.F. Lodish. 2000. Generation of mammalian cells stably expressing multiple genes at predetermined levels. Anal. Biochem. 280:20-28.

39. Masuda-Robens, J.M., S.N. Kutney, H. Qi, and M.M. Chou. 2003. The TRE17 oncogene encodes a component of a novel effector pathway for Rho GTPases Cdc42 and Rac1 and stimulates actin remodeling. Mol. Cell. Biol. 23:2151-2161. 